\title{
Human $\mathrm{ClCa} 1$ modulates anionic conduction of calcium-dependent chloride currents
}

\author{
Martine Hamann ${ }^{1,2}$, Adele Gibson ${ }^{2}$, Noel Davies ${ }^{1}$, Amanda Jowett ${ }^{2}$, Jean Philippe Walhin ${ }^{2}$, \\ Leanne Partington ${ }^{2}$, Karen Affleck ${ }^{2}$, Derek Trezise ${ }^{2}$ and Martin Main ${ }^{2}$ \\ ${ }^{1}$ Department of Cell Physiology and Pharmacology, Leicester University, Leicester LE1 9HN, UK \\ ${ }^{2}$ GlaxoSmithKline, Gunnels Wood Road, Stevenage SG1 2NY, UK
}

Proteins of the CLCA gene family including the human ClCal (hClCa1) have been suggested to constitute a new family of chloride channels mediating $\mathrm{Ca}^{2+}$-dependent $\mathrm{Cl}^{-}$currents. The present study examines the relationship between the hClCal protein and $\mathrm{Ca}^{2+}$-dependent $\mathrm{Cl}^{-}$ currents using heterologous expression of hClCal in HEK293 and NCIH522 cell lines and whole cell recordings. By contrast to previous reports claiming the absence of $\mathrm{Cl}^{-}$currents in HEK293 cells, we find that HEK293 and NCIH522 cell lines express constitutive $\mathrm{Ca}^{2+}$-dependent $\mathrm{Cl}^{-}$currents and show that hClCal increases the amplitude of $\mathrm{Ca}^{2+}$-dependent $\mathrm{Cl}^{-}$currents in those cells. We further show that hClCal does not modify the permeability sequence but increases the $\mathrm{Cl}^{-}$conductance while decreasing the $G_{\mathrm{SCN}^{-}} / G_{\mathrm{Cl}^{-}}$conductance ratio from $\sim 2-3$ to $\sim 1$. We use an Eyring rate theory (two barriers, one site channel) model and show that the effect of hClCal on the anionic channel can be simulated by its action on lowering the first and the second energy barriers. We conclude that hClCal does not form $\mathrm{Ca}^{2+}$-dependent $\mathrm{Cl}^{-}$ channels per se or enhance the trafficking/insertion of constitutive channels in the HEK293 and NCIH522 expression systems. Rather, hClCa1 elevates the single channel conductance of endogenous $\mathrm{Ca}^{2+}$-dependent $\mathrm{Cl}^{-}$channels by lowering the energy barriers for ion translocation through the pore.

(Received 31 January 2009; accepted after revision 20 March 2009; first published online 23 March 2009)
Corresponding author M. Hamann: Leicester University, Department of Cell Physiology and Pharmacology, Medical
Sciences Building, PO Box 138, University Road, Leicester LE1 9HN, UK. Email: mh86@le.ac.uk

Abbreviations $\mathrm{Br}^{-}$, bromide; $\mathrm{Cl}^{-}$, chloride; EDTA, ethylenediaminetetraacetic acid; $\mathrm{Glc}^{-}$, gluconate; GAPDH, glyceraldehyde 3-phosphate dehydrogenase; EGTA, glycol tetraacetic acid; HEK293, human embryonic kidney 293 cells; hClCa1, human ClCa1; HEDTA, hydroxyethylethylenediaminetriacetic acid; $\mathrm{I}^{-}$, iodide; Ise ${ }^{-}$, isethionate; $\mathrm{mClCa}$, murine ClCa3; NTA, nitrilotriacetic acid; NMDG, $\mathrm{N}$-methyl-D-glucamine chloride; pClCa1, porcine $\mathrm{ClCa1}$; $\mathrm{SCN}^{-}$, thiocyanate.

Three molecularly distinct $\mathrm{Cl}^{-}$channel families (CLC, CFTR, and ligand-gated GABA and glycine receptors) are well established whereas other gene families ( $\mathrm{ClCa}$ ) were also reported to encode $\mathrm{Cl}^{-}$channels but are less well characterized (Jentsch et al. 2002; Eggermont, 2004; Hartzell et al. 2005; Loewen \& Forsyth, 2005). The human $\mathrm{ClCa}$ protein (hClCal) is strongly up-regulated in mucus-producing epithelium of cystic fibrosis and asthmatic patients (Hoshino et al. 2002; Toda et al. 2002; Hauber et al. 2003, 2004), therefore generating great interest in its clinical relevance. The hClClal protein was initially proposed to be a $\mathrm{Ca}^{2+}$-dependent $\mathrm{Cl}^{-}$ channel. Evidence for this came from its heterologous expression in a range of cellular systems, which resulted in membrane currents activated in $\mathrm{Cl}^{-}$-selective conditions and with high internal $\mathrm{Ca}^{2+}$ concentrations. Currents were also blocked with chloride channel blockers (Gandhi et al. 1998; Gruber et al. 1998). More recently, Gibson et al. (2005) expressed hCLCA1 in HEK293 cells and showed that hCLCA1 is constitutively secreted in the extracellular medium. The N-terminal cleavage products of hCLCA1 could also be detected in bronchoalveolar lavage fluid taken from asthmatic subjects demonstrating release from cells in a pathological setting (Gibson et al. 2005). A bioinformatics analysis did not support the notion of transmembrane domains, further indicating that hCLCA1 is a non-integral membrane protein and is not a chloride channel per se. So far, electrophysiological data do not distinguish between $\mathrm{ClCa} 1$ family members being modulators or ion channels themselves. Studies with pClCal, the porcine ortholog of $\mathrm{hClCal}$, suggest that $\mathrm{ClCa} 1$ are chloride channel modulators that increase the 
amplitude of endogenous $\mathrm{Ca}^{2+}$-dependent $\mathrm{Cl}^{-}$currents (Loewen et al. 2002, 2003, 2004). Even in light of this evidence, questions still remain as to how members of the $\mathrm{ClCa}$ family are responsible for the increase of the amplitude of the chloride current. The present study seeks to shed new light on the relationship between the hClCal protein and the $\mathrm{Ca}^{2+}$-dependent $\mathrm{Cl}^{-}$channel by quantifying separate facets of the anion permeation process in cell lines expressing hClClal. We found that HEK293 and NCIH522 cell lines express constitutive $\mathrm{Ca}^{2+}$-dependent chloride currents. When expressed in those two cell lines, hClCal increased the conductance of $\mathrm{Ca}^{2+}$-dependent $\mathrm{Cl}^{-}$currents while leaving the relative permeability of other anions unaffected. We used a rate theory analysis to develop a model that describes the alteration of anion selectivity in the presence of hClCal and showed that the increase of the $\mathrm{Ca}^{2+}$-dependent $\mathrm{Cl}^{-}$ conductance in the presence of hClCa1 is due to its effect on the energy barriers that impact on the ion translocation event.

\section{Methods}

\section{Cell culture}

HEK293 and NCIH522 (a human lung epithelial adenocarcinoma cell line) were grown in Dulbecco's modified Eagle's medium (Sigma-Aldrich Company Ltd, Dorset, UK), supplemented with $10 \%$ heat-inactivated fetal bovine serum; $1 \%$ penicillin, streptomycin and glutamine; and $1 \%$ non-essential amino acids (all from Invitrogen Ltd, Paisley, UK). HEK293 cells stably expressing hClCA1 (HEK-hClCA1) were cultured in the presence of $0.8 \mathrm{mg} \mathrm{ml}^{-1}$ geneticin. Cells were maintained at $37^{\circ} \mathrm{C}$ in a humidified environment containing 5\% $\mathrm{CO}_{2}$ and split twice a week using Versene (Invitrogen), a solution containing $0.2 \mathrm{~g} \mathrm{l}^{-1} \mathrm{Na}_{4}$-EDTA in phosphate-buffered saline for $6-7 \mathrm{~min}$ at $37^{\circ} \mathrm{C}$.

\section{Cell lines}

For generation of the HEK293 clones stably expressing hClCa1, pCIN5-hClCa1 was transfected into HEK293 cells (American Type Culture Collection, Manassas, VA, USA). pCIN5_pl-hClCa1 DNA $(0.8 \mu \mathrm{g})$ was transfected into HEK293 using the lipofectamine 2000 protocol (Invitrogen). Neomycin-resistant colonies of cells were subsequently isolated by ring cloning and expanded under G418 (Gibco-Invitrogen) selection at $800 \mu \mathrm{g} \mathrm{ml}^{-1}$, and $\mathrm{hClCa}$ protein expression was confirmed by immunocytochemistry and Western blotting techniques using the A48 antibody directed against hCLCA1 as described below.

\section{Transient transfections}

Cells were cultured the day before transfection and plated on coverslips to achieve $\sim 70-80 \%$ confluency within $24 \mathrm{~h}$. Prior to transfection, cells were washed in serum-free Optimem medium (Gibco-Invitrogen). A first step consisted of adding the reagents $\left(0.8 \mu \mathrm{g} \mathrm{ml}^{-1}\right.$ pEGFP-N1 (BD Biosciences, Cat. no. 6085-1 (GenBank Accession no. U55762) with $7.2 \mu \mathrm{g} \mathrm{ml}^{-1}$ pcDNA3.1-hClCa1 or pcDNA3.1(+)) to Optimem, in a polypropylene tube and incubating the mixture for $10 \mathrm{~min}$ at room temperature. During the incubation period, $1 \%(\mathrm{v} / \mathrm{v})$ lipofectamine was mixed to Optimem medium in another tube. A second step consisted of combining the contents of both tubes and incubating the final mixture at room temperature for an additional $20 \mathrm{~min}$. Cells were transfected with this final mixture containing $0.4 \mu \mathrm{g} \mathrm{ml}^{-1} \mathrm{GFP}$ and $3.6 \mu \mathrm{g} \mathrm{ml}^{-1}$ of either hClCa1 or pcDNA3.1(+) for a $12-18 \mathrm{~h}$ period at $37^{\circ} \mathrm{C}$ before binding and function were assessed.

\section{Plasmids}

pcDNA3.1-D-V5-His-TOPO-CACC1 (hClCa1).

pcDNA3.1(+) was purchased from Invitrogen. Nested PCR primers were designed either side of the CACC1 gene sequence (PubMed Accession no. AF127036). Two rounds of nested PCR were necessary to obtain product from the colon template. The insert contains the entire CACC1 gene (with a short Kozak sequence prior to the initiator (i.e. caccATG) and a stop codon followed by one further codon at the very end). The product also contains a single silent (Val-Val) PCR mutation (base 645 gtT-gtA) in the coding sequence.

pCIN5_pl-hClCa1. The hClCal gene from pcDNA3.1D-V5-His-TOPO-CACC1 (hClCa1) was subcloned into the mammalian cell expression plasmid pCIN5_pl to give pCIN5_pl-hClCal. This plasmid contained the cytomegalovirus promoter and an internal ribosome entry site which allowed bicistronic expression of both hClCal and neomycin. As no compatible restriction sites were available for sub-cloning, a compatible Not $\mathrm{I}$ site was engineered into the pcDNA3.1-D-V5-His-TOPO-CACC1 (hClCa1) plasmid by site-directed mutagenesis. A pair of mutagenic primer oligonucleotides complementary to the pcDNA3.1 vector was synthesised. The site-directed mutagenesis was carried out according to the manufacturer's protocol (QuikChange Site-Directed Mutagenesis Kit, StratageneAgilent Technologies, TX, USA). The ClCa gene was then subcloned into the pCIN5_pl vector using these NotI sites and restriction enzyme analysis allowed for successfully subcloned plasmids to be selected. 


\section{Immunocytochemical staining procedure}

The A48 anti-hClCa1 antibody is a rabbit polyclonal antibody raised against the peptide sequence (GVNAARRRVIPQQSG), corresponding to amino acid residues 703-717 in $\mathrm{hClCa}$. Fluorescently tagged secondary antibodies were goat anti-rabbit IgG-Alexa568, and goat anti-rabbit IgG-Alexa488 (from Molecular Probes-Invitrogen). Live cell surface staining was carried out on cells grown on coverslips. Cells were incubated at $4^{\circ} \mathrm{C}$ for $15 \mathrm{~min}$, in incubation buffer (Hank's balanced salt solution-1\% bovine serum albumin-0.1\% sodium azide). Primary antibody incubations were carried out in incubation buffer, for $20 \mathrm{~min}$. Cells were washed twice and incubated with fluorescently tagged secondary antibodies, for $30 \mathrm{~min}$. Cells were then washed, fixed in $2 \%$ paraformaldehyde, mounted and examined on a Leica TCS-4D confocal microscope (Leica Micosystem UK Ltd, Milton Keynes, UK). The A48 antibody was used at $1 \mu \mathrm{g} \cdot \mathrm{ml}^{-1}$. The immunizing peptides were used as controls, to neutralize the antibodies, following a $15 \mathrm{~min}$ pre-incubation of antibody and peptide.

\section{Western blot analysis}

Cells were lysed in RIPA buffer (Upstate Biotech-Millipore UK Ltd, Watford, UK) containing $50 \mathrm{~nm}$ Tris- $\mathrm{HCl}, \mathrm{pH}$ 7.4, $150 \mathrm{~mm} \mathrm{NaCl}, 0.25 \%$ deoxycholic acid, 1\% NP-40 and $1 \mathrm{~mm}$ EDTA. Each sample $(10 \mu \mathrm{g})$ was run on NuPage 4-12\% polyacrylamide Bis-Tris gels (Invitrogen). Proteins were electrophoretically transferred to nitrocellulose membranes (Invitrogen) Membranes were blocked in 5\% non-fat milk and in Tris-buffered saline with 1\% Tween 20 and probed with the affinity-purified antibody A48 used at $0.5 \mu \mathrm{g} \cdot \mathrm{ml}^{-1}$. Following incubation with a horseradish peroxidase-conjugated anti-rabbit secondary antibody (Calbiochem-EMD Chemicals, Inc., NJ, USA), bands were visualised using enhanced chemiluminescence detection reagents (Amersham Biosciences, Buckinghamshire, $\mathrm{UK})$.

\section{Flow cytometry}

HEK293 cells $\left(5 \times 10^{5}\right)$ were washed in phosphatebuffered saline, containing 2.5\% FCS and $0.05 \%$ sodium azide, incubated with $0.55 \mu \mathrm{g}$ A48 antibody, or A48 and blocking peptide, for $10 \mathrm{~min}$ at room temperature. Cells were washed, incubated with anti-rabbit IgG-PE (Becton Dickinson Ltd, Plymouth, UK), at a dilution of $1: 50$, for $10 \mathrm{~min}$. Cells were washed, resuspended and immediately analysed on a Beckman Coulter EPICS XL-MCL FACS machine using EXPO32 ADC software (Beckman Coulter Ltd, High Wycombe, UK).

\section{TaqMan real time reverse transcriptase polymerase chain reaction}

TaqMan quantitative PCR was used to measure the mRNA expression levels for three hClCa protein subtypes (hClCa1, 2 or 4$)$ and two housekeeping genes ( $\beta$-actin and GAPDH).

\section{Generation of samples for TaqMan mRNA analysis}

Total RNA was prepared from up to $10^{7}$ cells from selected human cell lines (HEK293 and NCIH522) using the SV96 Total RNA isolation System (Promega, Madison, WI, USA). The total RNA samples were quantitfied using the RiboGreen fluorescent method (Molecular Probes, Eugene, OR, USA). The absence of genomic DNA contamination of the RNA samples was confirmed using B-actin TaqMan PCR. RNA ( $2 \mu \mathrm{g}$ ) was reverse transcribed using the High Capacity cDNA Archive Kit according to manufacturer's instructions (Applied Biosystems, Foster City, CA, USA). Each cDNA prepared was diluted to a concentration of $10 \mathrm{ng} \cdot \mathrm{ul}^{-1}$ and placed into two wells of a 384-well plate. Each well contained the cDNA produced from 20 ng total RNA from the appropriate cell line. All 384 -well plates were stored at $-80^{\circ} \mathrm{C}$ prior to use.

\section{TaqMan PCR conditions}

An $8 \mu \mathrm{l}$ volume of a PCR mix (containing $1 \mu \mathrm{l}$ TaqMan buffer, $2.4 \mu \mathrm{l} 25 \mathrm{mM}^{2} \mathrm{Mgl}_{2}, 0.2 \mu \mathrm{l}$ of $10 \mathrm{mM} \mathrm{dATP}$, $0.2 \mu \mathrm{l}$ of $20 \mathrm{~mm}$ dUTP, $0.2 \mu \mathrm{l}$ of $10 \mathrm{~mm} \mathrm{dCTP,} 0.2 \mu \mathrm{l}$ of $10 \mathrm{~mm}$ dGTP, $0.1 \mu \mathrm{l}$ uracil- $N$-glycosylase, $0.4 \mu \mathrm{l}$ of $10 \mu \mathrm{M}$ forward primer, $0.4 \mu \mathrm{l}$ of $10 \mu \mathrm{M}$ reverse primer, $0.2 \mu \mathrm{l} 5 \mu \mathrm{M}$ fluorogenic TaqMan probe, $0.05 \mu \mathrm{l}$ TaqGold (Applied Biosystems, Warrington, UK), $2.65 \mu$ l water) was added to each well using Biomek FX robotics (Beckman Coulter Ltd, High Wycombe, UK) and the plate was sealed using an Optical Adhesive Cover (Applied Biosystems, Warrington, UK). The PCR reaction was carried out on an ABI7900HT Sequence Detection System (Applied Biosystems, Warrington, UK) using the PCR parameters: $50^{\circ} \mathrm{C}$ for $2 \mathrm{~min}, 95^{\circ} \mathrm{C}$ for $10 \mathrm{~min}$ and 45 cycles of $95^{\circ} \mathrm{C}$ for $15 \mathrm{~s}, 60^{\circ} \mathrm{C}$ for $1 \mathrm{~min}$. The level of mRNA-derived cDNA in each sample was calculated from the TaqMan signal using duplicate human genomic DNA calibration standards included in each. Table 1 describes the gene-specific reagents (forward primer, reverse primer and TaqMan probe).

\section{Patch clamp electrophysiology}

Whole cell currents were recorded at room temperature $\left(20-23^{\circ} \mathrm{C}\right)$ using an Axopatch 200B (Axon Instruments, Foster City, CA, USA). Data acquisition and analysis 
Table 1. Gene-specific primers/probes used for TaqMan RT-PCR analysis

\begin{tabular}{lccl}
\hline Gene & GenBank acc. no. & Primer & \multicolumn{1}{c}{ Sequence 5'-3' } \\
\hline hClCa1 & NM_001285 & S & GATGTCCCAAATGCTCCCATAC \\
& & AS & CCCAGGAGCTGTCCAAGTCA \\
& & ATCTCTTCCCACCTGGCCAAATCACC \\
hClCa2 & NM_006536 & S & TCGGACAGCTAGTCTTTGGATTC \\
& & AS & TGATGGGTATTGTTCAGGGTGTAA \\
& & P & AGGAACAGCTAAGCCTGGGCACTGG \\
hClCa4 & NM_012128 & S & CTTGGAGGATTTCAGCCGAA \\
& & AS & AGGGAAGGCTTGGGACTTGT \\
& & P & AGCATCCGGAGGTGCATTTGTGGTA \\
B actin & X00351 & S & GAGCTACGAGCTGCCTGAACG \\
& & AS & GTAGTTTCGTGGATGCCACAGGACT \\
& & P & CATCACCATTGGCAATGAGCGGTTCC \\
GAPDH & XM006959 & S & CAAGGTCATCCATGACAACTTTG \\
& & AS & GGGCCATCCACAGTCTTCTG \\
& & P & AACACAGTCCATGCCATCACTGCCA \\
\hline
\end{tabular}

$S$, sense forward primer; AS, antisense reverse primer; $P$, Taqman probe; $n$, nucleotide position relative to first ATG start codon of coding sequence. Primer and probe sequences were designed using Primer Express software (Version 2.0, Applied Biosystems) according to the manufacturer's guidelines. Each TaqMan probe was synthesised with the fluorescent reporter dye FAM (6-carboxy-fluorescein) attached to the $5^{\prime}$-end and a quencher dye TAMRA (6-carboxy-tetramethyl-rhodamine) to the $3^{\prime}$-end.

were performed via a Digidata 1320 interface (Axon Instruments) using pClamp 9 software (Axon Instruments). After formation of a gigaohm seal and establishment of whole cell access, cells were voltage clamped at $-80 \mathrm{mV}$. Membrane capacitance was calculated from the integral of the current transient in response to $10 \mathrm{mV}$ hyperpolarizing pulses. The series resistance did not exceed $10 \mathrm{M} \Omega$ and was compensated by $70-75 \%$. Records were sampled at between $5-10 \mathrm{kHz}$ and filtered at $1-2 \mathrm{kHz}$ with a four-pole Bessel filter. Current-voltage relationships were examined using $600 \mathrm{~ms}$ voltage steps from a holding potential of $-80 \mathrm{mV}$ to potentials from $-100 \mathrm{mV}$ to $+80 \mathrm{mV}$ at $20 \mathrm{mV}$ intervals. Ramp currents were examined from a holding potential of $-80 \mathrm{mV}$ to potentials from $-100 \mathrm{mV}$ to $100 \mathrm{mV}$ over $1 \mathrm{~s}$.

\section{Solutions}

Cultured cells plated on glass coverslips were transferred to the stage of an inverted microscope and superfused with an extracellular solution at a rate of $2-3 \mathrm{ml} . \mathrm{min}^{-1}$. Solutions changes were performed using a series of gravitionally fed tubes and valves.

\section{Calcium-dependent chloride currents}

The standard extracellular solution contained (in $\mathrm{mM}$ ): $\mathrm{NaCl}$ (126), Hepes (10), sucrose (30), $\mathrm{CaCl}_{2}$ (2) and
$\mathrm{MgCl}_{2}$ (2). The $\mathrm{pH}$ was set to 7.4 with $\mathrm{NaOH}$. To quantify the chloride current, $\mathrm{a} \mathrm{Cl}^{-}$-free external solution was used where gluconate replaced the $\mathrm{Cl}^{-}$in the standard external solution (using sodium gluconate, calcium gluconate and magnesium gluconate at the appropriate concentrations). In chloride substitution experiments described in Fig. 7, $126 \mathrm{mM}$ external $\mathrm{Cl}^{-}$ $\left(\mathrm{Cl}^{-}{ }_{\mathrm{o}}\right)$ was replaced by equimolar bromide $\left(\mathrm{Br}^{-}\right)$, iodide $\left(\mathrm{I}^{-}\right)$, thiocyanate $\left(\mathrm{SCN}^{-}\right)$, isethionate $\left(\mathrm{Ise}^{-}\right)$or gluconate $\left(\mathrm{Glc}^{-}\right)$. Patch pipettes (borosilicate glass) were pulled using a Sutter P-97 pipette puller (Instrument Company, Novato, CA, USA) and filled with a solution composed of (in mM): $\mathrm{N}$-methyl-D-glucamine chloride (120), $\mathrm{CaCl}_{2}$ (4.36), $\mathrm{MgCl}_{2}$ (2), HEDTA (8) and Hepes (10), $\mathrm{pH} 7.1$. We quantified the response at steady state by buffering and estimating the actual free intracellular concentration (as described in Kuruma \& Hartzell, 2000). The concentration of free $\mathrm{Ca}^{2+}$ in this solution was calculated to be $10 \mu \mathrm{M}$ using the software WinMAXC (v.2.05; Chris Patton, Stanford, CA, USA). In the experiments where different $\left[\mathrm{Ca}^{2+}\right]_{\mathrm{i}}$ were used, the pipette solution contained: (a) $1.69 \mathrm{mM} \mathrm{CaCl}_{2}$ and $5 \mathrm{~mm} \mathrm{EGTA;} \mathrm{(b)} 3.95 \mathrm{mM} \mathrm{CaCl}_{2}$ and $5 \mathrm{~mm}$ EGTA; (c) $1.87 \mathrm{mM} \mathrm{CaCl}_{2}$ and $5 \mathrm{~mm} \operatorname{HEDTA}$; (d) $1.47 \mathrm{mM} \mathrm{CaCl} 2$ and $5 \mathrm{~mm}$ nitrilotriacetic acid (NTA); (e) $3.35 \mathrm{mM} \mathrm{CaCl}_{2}$ and $5 \mathrm{~mm} \mathrm{NTA}$ to give a final free $\left[\mathrm{Ca}^{2+}\right]_{\mathrm{i}}$ of: (a) $130 \mathrm{nM}$, (b) $1 \mu \mathrm{M}$, (c) $5 \mu \mathrm{M}$, (d) $100 \mu \mathrm{M}$ and (e) $486 \mu \mathrm{M}$, respectively. Osmolarity was adjusted to 295 mosmol $^{-1}$ with sucrose. Currents elicited by 1 or $2 \mathrm{mM}\left[\mathrm{Ca}^{2+}\right]_{\mathrm{i}}$ were recorded with a pipette solution 
described by Gruber et al. (1998) and Britton et al. (2002) that contained (in mM): NMDG-Cl (112), sucrose (30), Hepes (5), $\mathrm{MgCl}_{2}$ (2) and $\mathrm{CaCl}_{2}$ (1) or (2).

\section{Data analysis}

Rectification ratios were calculated as the current amplitude at $+80 \mathrm{mV}$ divided by the current amplitude at $-80 \mathrm{mV}$.

Permeability ratios were calculated using the Goldman-Hodgkin-Katz equation as follows:

$$
P_{\text {sub }} / P_{\mathrm{Cl}}=\left\{\exp \left(\frac{\Delta E_{\text {rev }}}{R T / z F}\right)-\left(\frac{[\mathrm{Cl}]_{0}}{[\mathrm{Cl}]_{0}^{\prime}}\right)\right\} \cdot\left(\frac{[\mathrm{Cl}]_{0}^{\prime}}{[\mathrm{sub}]_{0}}\right)
$$

where $\Delta E_{\text {rev }}$ is the measured shift in zero current (reversal) potential after $\mathrm{Cl}_{0}$ is replaced with the substitute ion sub $[\mathrm{Cl}]_{0}^{\prime}$ is the bath concentration of $\mathrm{Cl},[\mathrm{Cl}]_{0}$ is the residual $\mathrm{Cl}$ in the substituted solution, [sub $]_{0}$ is the concentration of the substitute ion and $R, T, z$ and $F$ are the universal gas constant, the absolute temperature, the valency and the Faraday constant, respectively.

Conductance $(g)$ was calculated as follows:

$$
g=\left(\frac{I}{E_{\mathrm{m}}-E_{\text {rev }}}\right)
$$

where $E_{\mathrm{m}}$ is the membrane voltage, $I$ the measured current and $E_{\text {rev }}$ the zero current potential. The conductance was estimated at a stepping potential of $+80 \mathrm{mV}$ and the conductance of an anion $\mathrm{X}$ relative to that of $\mathrm{Cl}^{-}$ $\left(G_{X} / G_{\mathrm{Cl}^{-}}\right)$was estimated as the conductance ratio under bi-anionic conditions.

Concentration-response curves relating the free internal calcium $\left[\text { free } \mathrm{Ca}^{2+}\right]_{\mathrm{i}}$ to the activation of the chloride current were fitted in Origin 5.0 software, according to a non-linear logistic function as follows:

$$
y=\left(\frac{\left(A_{1}-A_{2}\right)}{1+\left(x / x_{0}\right)^{p}}\right)+A_{2}
$$

where $x_{0}\left(\mathrm{EC}_{50}\right)$ is the mid-dose effect, $p$ is the slope, $A_{1}$ and $A_{2}$ are the predicted responses to a 0 and an infinite [free $\mathrm{Ca}^{2+}$ ], respectively. Fit estimates were calculated using the least square algorithm.

\section{Statistics}

All data are means \pm S.D. of $n$ individual cells. A Student's $t$ test was used to compare data and results were considered significant when $P<0.05$.

\section{Modelling channel permeation}

The channel was modelled assuming a single binding site, $X$, that could be either empty or occupied by a single ion. Thus, in the presence of two permeant ions, $\mathrm{A}$ and $\mathrm{B}$, the following scheme indicates the transitions considered, where the rates are represented by lower case letters and the subscripts $o$ and $i$ indicate external and internal, respectively:

$$
\begin{aligned}
& X+\mathrm{A}_{\mathrm{o}} \underset{a_{-1}}{\stackrel{a_{1}}{=}} X \mathrm{~A} \underset{a_{-2}}{\stackrel{a_{2}}{=}} X+\mathrm{A}_{\mathrm{i}} \\
& X+\mathrm{B}_{\mathrm{o}} \underset{b_{-1}}{\stackrel{b_{1}}{=}} X \mathrm{~B} \underset{b_{-2}}{\stackrel{b_{2}}{=}} X+\mathrm{B}_{\mathrm{i}}
\end{aligned}
$$

At equilibrium, $\mathrm{d}(X \mathrm{~A}) / \mathrm{d} t=0$ and $\mathrm{d}(X \mathrm{~B}) / \mathrm{d} t=0$, thus:

$$
\begin{aligned}
& P_{X} a_{1}+P_{X} a_{-2}=P_{X A} a_{-1}+P_{X A} a_{2} \\
& P_{X} b_{1}+P_{X} b_{-2}=P_{X B} b_{-1}+P_{X B} b_{2}
\end{aligned}
$$

Furthermore,

$$
P_{X}+P_{X \mathrm{~A}}+P_{X \mathrm{~B}}=1
$$

where $P_{X}$ denotes the probability of being in state $X$ (unoccupied binding site) and so on. By solving these simultaneous equations, expressions for the probabilities, $P_{X}, P_{X \mathrm{~A}}$ and $P_{X \mathrm{~B}}$ in terms of the rates can be derived. The net flux of ions $\mathrm{A}$ and $\mathrm{B}, J_{\mathrm{A}}$ and $J_{\mathrm{B}}$, respectively, are then obtained from the difference in the absolute transition rates for efflux and influx:

$$
J_{\mathrm{A}}=P_{X \mathrm{~A}} a_{-1}-P_{X} a_{1} \text { and } J_{\mathrm{B}}=P_{X \mathrm{~B}} b_{-1}-P_{X} b_{1}
$$

The current carried by ion $\mathrm{A}$ is given by $i_{\mathrm{A}}=z e J_{\mathrm{A}}$ where $e$ is $1.6 \times 10^{-19} \mathrm{C}$. A similar argument is used for ion $\mathrm{B}$ and the total current will therefore be $i_{\mathrm{T}}=i_{\mathrm{A}}+i_{\mathrm{B}}$.

To calculate the rates, we used a rate theory model (Glässtone et al. 1941; Davies et al. 1996) in which the channel has two energy barriers and a single well or binding site able to accommodate a monovalent anion. For simplicity we distributed the barriers and binding site equally within the voltage field (see Scheme 1).

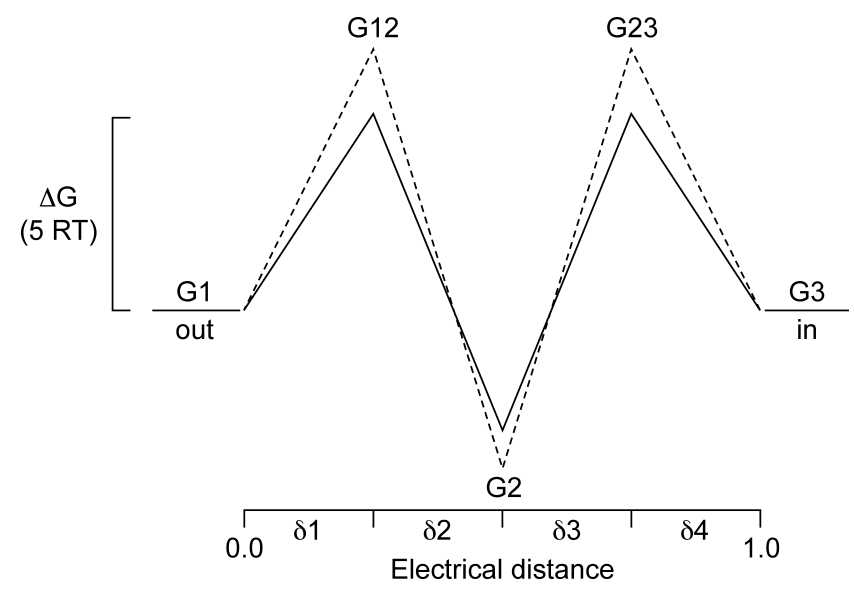

Scheme 1

The schematic representation of the energy profiles for ion $\mathrm{A}$ (continuous line) and ion $\mathrm{B}$ (dashed line) in a channel with two barriers (energy levels G12 and G23, 
where $G$ denotes Gibbs free energy) and one well (binding site, G2). The abscissa represents the positions of the barriers and well within the voltage field of the membrane, which, in our calculations, have been distributed equally, such that $\delta 1, \delta 2, \delta 3$ and $\delta 4=0.25$. The ordinate represents the difference in free energy levels $(\Delta G)$ in $R T$ units relative to those in the bulk solution. The well can only be occupied by one ion at a time.

An ion traversing from outside of the membrane to the inside must first 'hop' over barrier G1 to occupy the binding site and then 'hop' over barrier $G 23$ to reach the inside. The general rate constant, $k(V)$, for an ion hopping from one state to another is related to the energy difference between the two states, $G(V)$, by:

$$
k(V)=v \cdot \exp (-G(V) / R T)
$$

where $v=k_{\mathrm{B}} T / h k_{B}$ is Boltzmann's constant, $h$ is Planck's constant so $v$ has the dimensions of transitions per unit time. When calculating $G(V)$ an electrical term associated with an ion of valence $z$ moving through a voltage field needs to be included to the zero voltage energy difference $G(0)$. The direction of flow is important with the outward flow resulting in addition of the electrical component and inward flow to subtraction. Thus:

$$
G(V)_{\text {out }}=G(0)+z \delta F V \text { and } G(V)_{\text {in }}=G(0)-z \delta F V
$$

Here $\delta$ is the proportion of the voltage field (electrical distance) travelled by the ion for a particular jump and $z$ is the valence of the ion. If the values of the energy levels are in RT units then the rates for ion A moving from outside to inside will be given by:

$$
\begin{aligned}
& a_{1}=[\mathrm{A}]_{o} v \exp \left(-\mathrm{G} 12-z \delta_{1} F V / R T\right) \\
& a_{2}=v \exp \left(-(\mathrm{G} 23-\mathrm{G} 2)-z \delta_{3} F V / R T\right)
\end{aligned}
$$

where $R, T$ and $F$ have their usual thermodynamic meanings.

\section{Results}

\section{Detection of hClCal mRNA}

We screened for the presence of the hClCal gene in different HEK293 clones stably transfected with pCIN5_pl-hClCal DNA using a highly sensitive and quantitative PCR approach and proceeded to a quantitative analysis of the hClCal gene in those cell lines. Figure $1 A$ shows that $\mathrm{hClCal}$ gene expression is detected at different levels within different HEK293 clones stably transfected with pCIN5_pl-hClCal DNA, ranging from $1.4 \times 10^{6}$ to over $10.5 \times 10^{6}$ copies of mRNA detected per $50 \mathrm{ng}^{-1}$ mRNA pool for clone 6 and 4, respectively. By comparison, the hClCal gene is undetected in HEK293 wild type and in HEK293 stably transfected with the
pCIN5 vector with less than $1 \times 10^{3}$ copies of mRNA detected per $50 \mathrm{ng} \mathrm{mRNA}$ pool. The quantitative analysis of hClCa 2 and hClCa4 mRNAs was also performed in the same cell types and showed that by contrast to hClCa1 specifically expressed at high levels in HEK293 clones stably transfected with pCIN5_pl-hCLCA1 DNA, $\mathrm{hClCa} 4$ and hClCa2 mRNA was undetected in those cells, with less than $1 \times 10^{3}$ and $4 \times 10^{3}$ copies of mRNA per $50 \mathrm{ng}^{-1} \mathrm{mRNA}$ pool for hClCa4 and hClCa2, respectively. Those low expression levels are significantly different from control $\beta$ actin and GAPDH genes ranging from $0.5 \times 10^{6}$ to over $2 \times 10^{6}$ copies of mRNA detected per $50 \mathrm{ng}^{-1}$ mRNA pool (Fig. $1 B-D$ ). Similar experiments have also been performed in NCIH522 or HEK293 transiently transfected with GFP and pcDNA3.1(+)-hClCa1 in which we confirmed the presence of the hClCal (see Fig. $6 \mathrm{~A}$ for NCIH522).

\section{Immunological detection of hClCa1}

We used the flow cytometry technique to quantify the fluorescent labelling of hClCal on various clones of HEK-hClCal (Fig. 2). Whereas the labelling linked to wild type HEK293 (HEK293-WT) and HEK293 stably transfected with the pCIN5 vector was distributed around a single peak of low background fluorescence (Fig. 2A), clones of HEK293 cells transfected with hClCa1 were all emitting a higher level of fluorescence (Fig. $2 B-D$ ) with a variation in the fluorescence intensity and the number of labelled cells as summarized in Fig. 2E. Immunofluorescence labelling was absent when $\mathrm{HEK}-\mathrm{hClCa} 1$ cells were previously treated with the antibody A48 blocking peptide, consistent with the neutralization of the A48 antibody by the blocking peptide (shown for clones 4 and 8 in Fig. $2 C-D$ ). Two HEK293 clones stably transfected with pCIN5_pl-hClCa1 DNA (HEK-hClCa1 clones 4 and 8) were fractionated and examined by Western blot with the A48 antibody (Gibson et al. 2005, Fig. 3A). Both HEK-hClCa1 clones were found to contain both the full-length protein $(125 \mathrm{kDa})$ and the $\mathrm{N}$-terminal cleavage product of hClCal of $83 \mathrm{kDa}$ or $90 \mathrm{kDa}$ described in previous reports (Gruber et al. 1998; Gibson et al. 2005). Immunofluorescent labelling of hClCal using the A48 antibody revealed that hClCal was expressed at the cell surface of non-permeabilized HEK-hClCal clones 4 and 8 (Fig. $3 B$ ) or of NCIH522 cells (Fig. 6B). In this latter case, as expected, the A48 cell surface labelling also coincided with the green GFP-pcDNA3.1(+) hClCa1 cytoplasmic labelling. Electrophysiological studies were conducted on HEK-hClCal clones 4 and 8 in which $99 \%$ and $97 \%$ of cells, respectively, were labelled with the A48 antibody (Fig. 2E). 


\section{Presence of $\mathrm{Ca}^{2+}$-sensitive chloride currents in HEK293 and NCIH522 cells}

Before studying potential effects of hClCal on chloride currents in HEK293 or NCIH522 cells, we checked whether those cells displayed chloride currents in the absence of hClCa1. Intensity-voltage curves following step or ramp stimulations showed outwardly rectifying currents recorded from either wild type, vector or hClCa1 transfected HEK293 cells (Fig. $4 C$ and $G$ ) with a rectification ratio at around 2 (see Table 2 for summary). This was similar to the intensity-voltage curves described in HEK293 cells transfected with mouse ClCa1 (Gandhi et al. 1998). By stepping to $+80 \mathrm{mV}$, a fast decaying current with a time constant of around 100-200 ms was first observed. Eighty to ninety per cent of the remaining recorded current was steady state after a $1 \mathrm{~s}$ step (Fig. $4 A$ and E). Currents reversed at a potential close to the theoretical chloride equilibrium potential of $0 \mathrm{mV}$ (Table 2) and were abolished when external chloride was replaced with an equimolar concentration of impermeant anion gluconate (Fig. $4 B$ and $F$ ). In this latter case, the mean reversal potential shifted to positive potentials exceeding $25 \mathrm{mV}$ consistent with chloride currents. Chloride currents were inhibited by $300 \mu \mathrm{M}$ DIDS, $300 \mu \mathrm{M}$ SITS and $100 \mu \mathrm{M}$ niflumic acic (Table 2). We tested whether chloride currents were dependent on the internal free calcium concentration. Figure $5 B$ shows that chloride currents were dependent on varying the internal free calcium concentration from $130 \mathrm{~nm}$ to $486 \mu \mathrm{M}$. $\mathrm{EC}_{50}$ values of $21 \mu \mathrm{M}$ and $7 \mu \mathrm{M}$ were obtained for HEK293 wild type and PCIN5 vector transfected cells, respectively,
Figure 1. Taqman quantitative PCR analysis of $\mathrm{ClCa}$ mRNA protein subtypes in HEK293 cells

The mRNA is extracted from HEK293 wild type (WT) and HEK293 cells stably expressing PCIN5 (PCIN5) or pCIN5-hCCla1 (clonal cultures C2 to C8). Quantitative $\mathrm{PCR}$ analysis was performed for $A, \mathrm{hClCa} 1 ; B, \mathrm{hClCa}$; $C, \beta$ actin; $D$, GAPDH
A

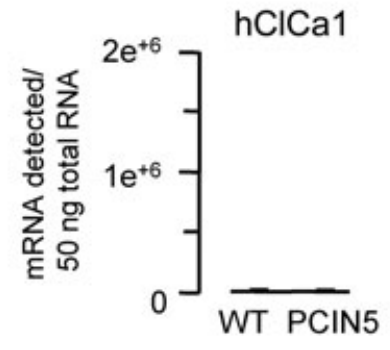

$B$

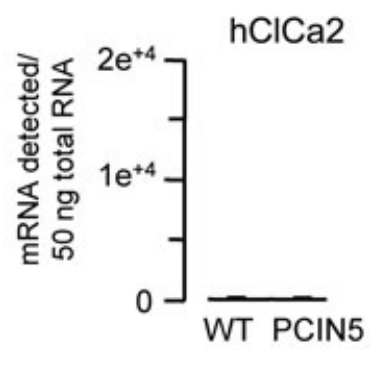

C

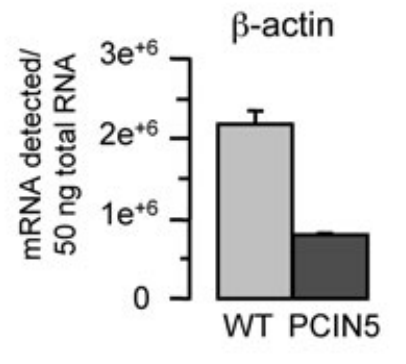

$D$

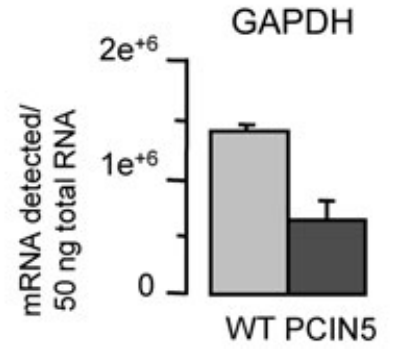

HEK 293
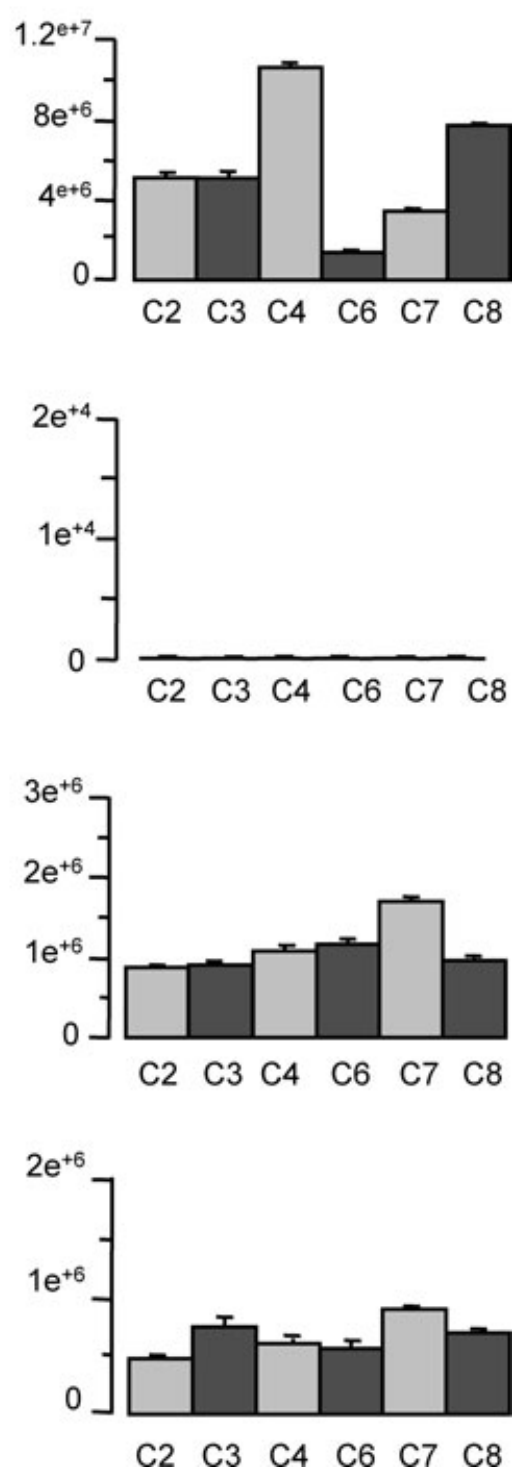
whilst values for HEK293 hClCal clones 4 and 8 were slightly lower ( $2 \mu \mathrm{M}$ and $3 \mu \mathrm{M}$, respectively). In previous studies on HEK293 cells (Gandhi et al. 1998; Gruber et al. 1998, 1999), $\mathrm{Ca}^{2+}$-dependent chloride currents required a high concentration of $\mathrm{Ca}^{2+}(1-2 \mathrm{mM})$ for activation.
When we used $1 \mathrm{mM} \mathrm{Ca}^{2+}$ in our intracellular medium (similar to Gandhi et al. 1998; Gruber et al. 1998, 1999), the quality of the whole cell recordings became critical after a few minutes of recording, indicating a disrupted membrane. Altogether, results indicate that all HEK293

HEK 293

A

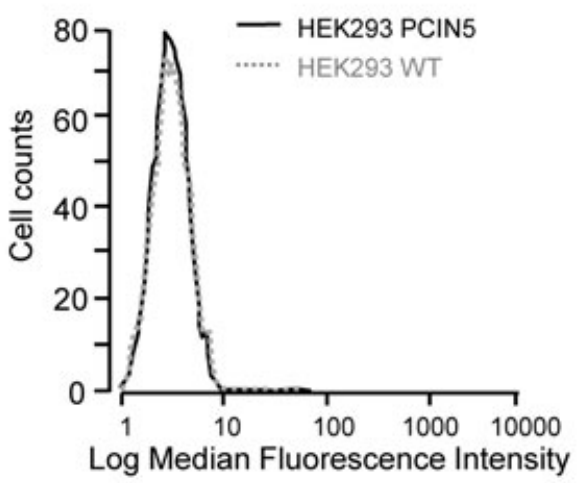

C

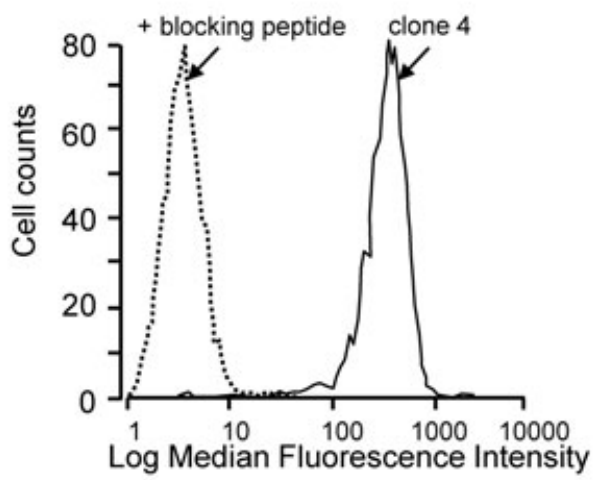

E

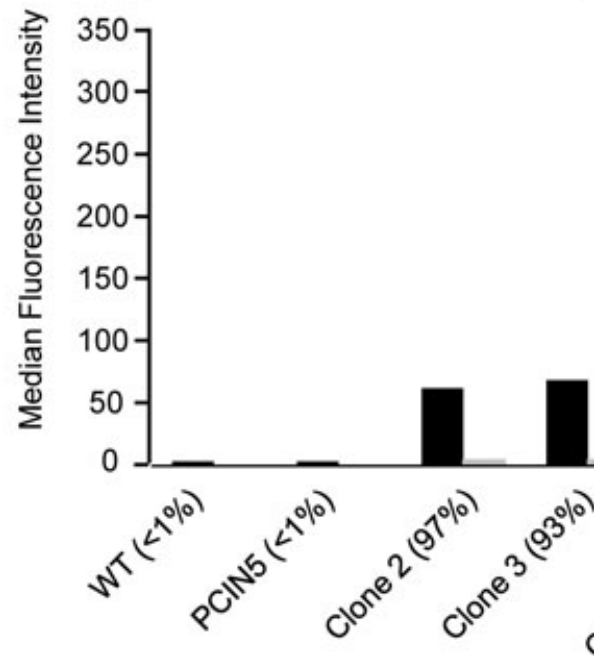

B

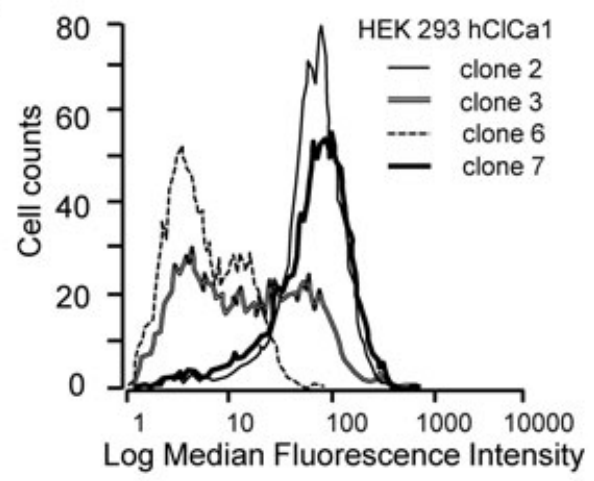

$D$

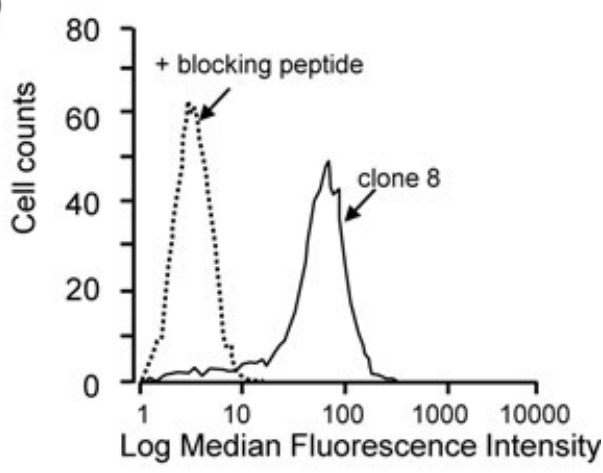

Figure 2. Flow cytometry quantitative analysis of the $\mathbf{A} 48$ fluorescent labelling

Distribution of the fluorescence intensity from A, HEK293 cells stably transfected with pCIN5 and HEK 293 wild type. B, HEK293 cells stably transfected with pCIN5-hCICa1 clones 2, 3, 6 and 7. C, HEK293 cells stably transfected with $\mathrm{pCIN5}$-hClCa1 clones 4 in control condition and in presence of the A48 blocking peptide. D, HEK293 cells stably transfected with pCIN5-hCICa1 clone 8 in control condition and in presence of the A48 blocking peptide. E, summary histograms showing the intensity of the A48 labelling in the different HEK293 populations in the absence and the presence of the blocking peptide. For each condition the percentage of labelled cells is in parentheses. 
cells, whether transfected or not with hClCal, display $\mathrm{Ca}^{2+}$-sensitive chloride currents with the internal free calcium concentration from $130 \mathrm{~nm}$ to $486 \mu \mathrm{M}$. Similar outwardly rectifying $\mathrm{Ca}^{2+}$-sensitive chloride currents were recorded in NCIH522 (Fig. 6C-H) or in HEK293 (not shown) that were transiently transfected with either pcDNA3(+) or hClCa1.

\section{hClCa1 increases the amplitude of $\mathrm{Ca}^{2+}$-sensitive chloride currents}

Whereas an equimolar substitution of gluconate abolished the outwardly rectifying chloride current, the inward current due to the exit of chloride stayed unaffected. This is reflected by the small effect of gluconate substitution on the holding current recorded at $-80 \mathrm{mV}$
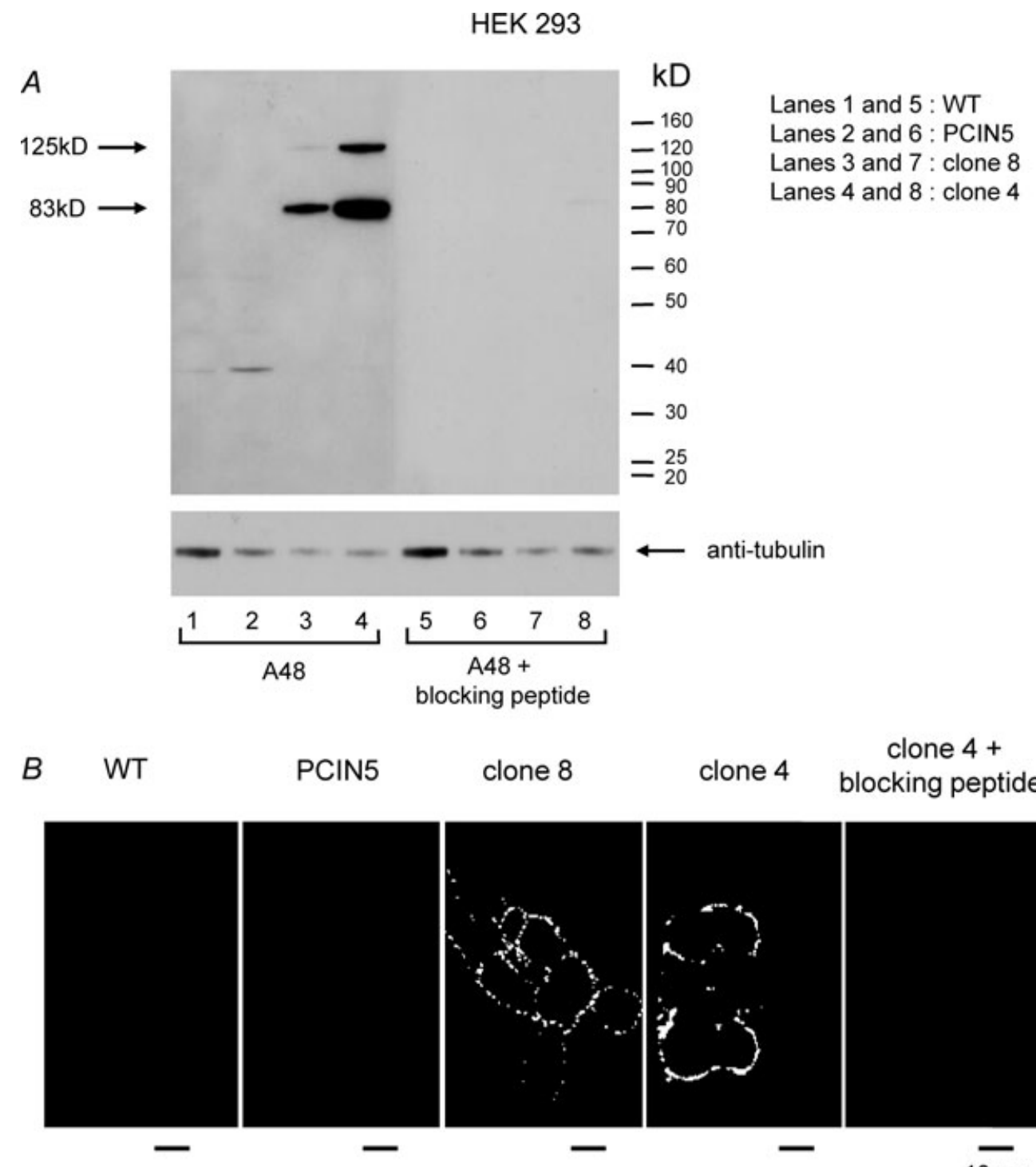

clone 8

clone 4

clone $4+$ blocking peptide
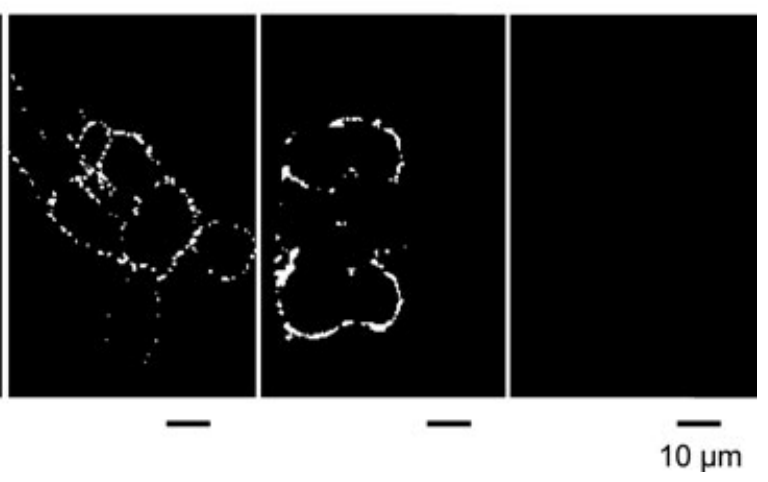

Figure 3. Western blot analysis and immunofluorescent labelling of hClCa1 with the A48 antibody A, fractionated HEK293 cells were examined by Western blot and probed with anti-hCLCA1 antibody (A48) to show the labelling of hClCa1 in HEK293 cells stably expressing pCIN5-hCCla1 (clones 4 and 8, lanes 3 and 4, respectively) by opposition to HEK293 wild type (WT) and HEK293 cells stably expressing PCIN5 (PCIN5) (lanes 1 and 2, respectively). Lanes 5 to 8 represent the negative control conditions (i.e. with the blocking peptide) for lanes 1 to 4 , respectively. $B$, immunofluorescence light microscopy showing that the A48 is labelling the cell surface of HEK293 cells stably expressing pCIN5-hCCla1 (clones 4 and 8) as opposed to HEK293 wild type (WT) and HEK293 cells stably expressing PCIN5 (PCIN5). No labelling is observed when pre-incubating HEK293 cells stably expressing pCIN5-hCCla1 clone 4 with the blocking peptide. 
Table 2. Similar properties of the calcium-dependent chloride current in HEK293 wild type and in HEK293 cells stably transfected with either PCIN5 or with PCIN5-hCICa1 (clones 4 and 8)

\begin{tabular}{lcccc}
\hline & HEK-WT & HEK-PCIN5 & $\begin{array}{c}\text { HEK-PCIN5- } \\
\text { hCICa1 clone 4 }\end{array}$ & $\begin{array}{c}\text { HEK-PCIN5- } \\
\text { hCICa1 clone 8 }\end{array}$ \\
\hline \% rectification & $2.2 \pm 2.8(9)$ & $2.1 \pm 3.1(7)$ & $1.9 \pm 2.7(9)$ & $2.2 \pm 1.9(9)$ \\
I steady state (\%) & $87 \pm 11(9)$ & $78 \pm 15(7)$ & $89 \pm 5(9)$ & $90 \pm 75(9)$ \\
Tau I transient (ms) & $141 \pm 49(5)$ & $118 \pm 103(7)$ & $100 \pm 85(5)$ & $180 \pm 170(4)$ \\
$E_{\text {rev }}$ (mV) & $-1 \pm 10(8)$ & $4 \pm 8(9)$ & $2 \pm 7(8)$ & $3 \pm 8(8)$ \\
$\%$ block (DIDS) & $94 \pm 14(6)$ & $90 \pm 9(8)$ & $98 \pm 3(5)$ & $99 \pm 1(4)$ \\
$\%$ block (SITS) & $65 \pm 27(4)$ & $44 \pm 18(7)$ & $48 \pm 28(6)$ & $41 \pm 18(6)$ \\
$\%$ block (Nif. acid) & $36 \pm 19(4)$ & $31 \pm 12(8)$ & $35 \pm 12(5)$ & $42 \pm 23(5)$ \\
\hline
\end{tabular}

The rectification was estimated as the current recorded at $+80 \mathrm{mV}$ divided by the current at $-80 \mathrm{mV}$. The steady state current was calculated as the proportion of remaining current recorded at the end of a $5 \mathrm{~s}$ pulse from $-80 \mathrm{mV}$ to $+80 \mathrm{mV}$ and the value of the decay was determined by fitting the transient current at the start of the pulse. The percentage of block by $300 \mu \mathrm{M}$ DIDS, $300 \mu \mathrm{M}$ SITS and $100 \mu \mathrm{M}$ niflumic acid was calculated at $+80 \mathrm{mV}$. Values are mean \pm S.D. (n, numbers).

\section{HEK293 PCIN5}

$A$

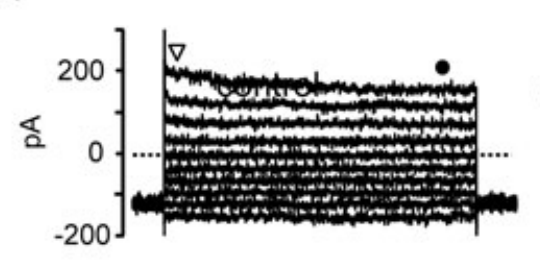

C

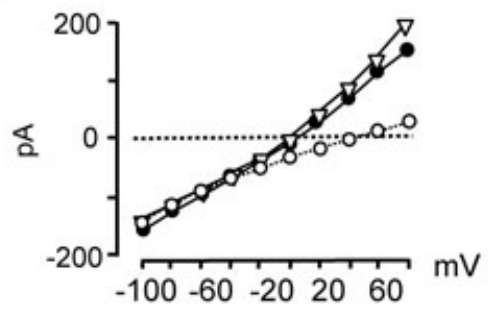

$B$

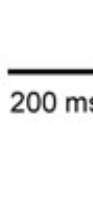

gluconate

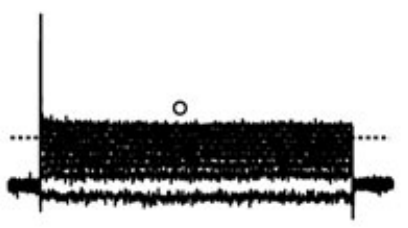

$D$

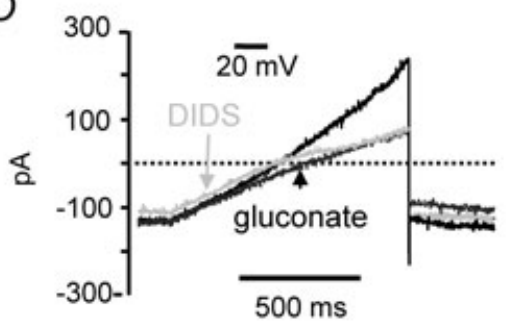

\section{HEK293-hClCa1}

E

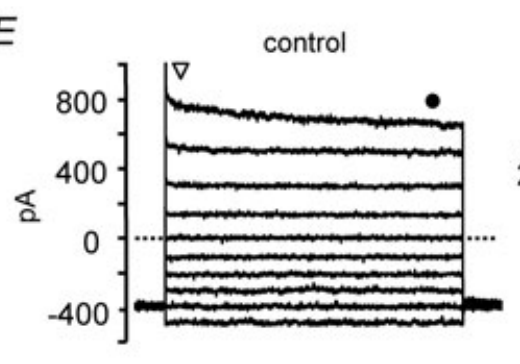

G

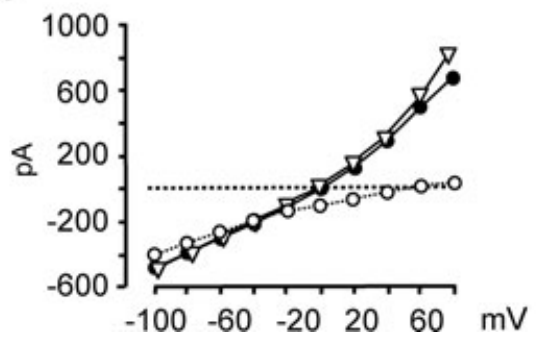

$F$

$200 \mathrm{~ms}$

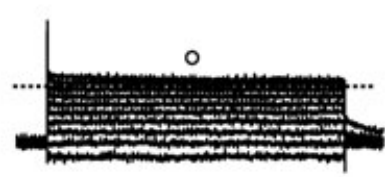

H

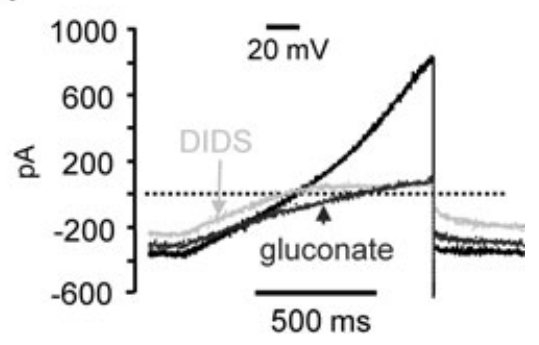

Figure 4. Whole cell recording of chloride currents in HEK293 cells

$A-D$ in HEK293 cells stably transfected with PCIN5 and $E-H$ in HEK293 cells stably transfected with PCIN5-hClCa1. $A$ and $E$ are representative whole cell currents in control conditions from a holding potential of $-80 \mathrm{mV}$ to $80 \mathrm{mV}$ ( $20 \mathrm{mV}$ steps). The first step is a hyperpolarising step to $-100 \mathrm{mV}$. $B$ and $F$ represent similar currents recorded after substituting external chloride with external gluconate. $C$ and $G$ represent the $I-V$ relationship of the currents measured at the beginning of the $\operatorname{step}(\nabla)$, at the end of the step $(\bullet)$ and after substituting chloride with gluconate (O). $D$ and $H$ are representative currents recorded during voltage ramp protocols from a holding potential of $-80 \mathrm{mV}$ to $+100 \mathrm{mV}$ in control condition (black), after substituting chloride with gluconate (arrow) and after perfusion of $300 \mu \mathrm{M}$ DIDS (grey). Note the difference in the amplitude scale bar between $A$ and $E$, between $C$ and $G$ and between $D$ and $H$. 
(Fig. $4 D$ and $H$ ). We used the ability of gluconate to substitute for external chloride in a quick and reversible manner to quantify the density of chloride currents at $+80 \mathrm{mV}$ in both HEK293 and NCIH522 cells. We found that hClCal was associated with an increase in the amplitude of the $\mathrm{Ca}^{2+}$-sensitive chloride HEK293 cells from $118 \pm 91 \mathrm{pA}(n=21)$ to $1033 \pm 861 \mathrm{pA}(n=13$, clone $4, P=0.0018)$. Examples are given in Fig. $4 E$; Fig. $6 E$ and results normalised to the cell capacitance are summarised in Figs $5 A$ and $6 G$ for HEK293 and NCIH522, respectively). Figure 5A shows that HEK293 wild type and HEK293 transfected with the PCIN5 vector express chloride currents at a density of $7 \pm 10$ pA.pF ${ }^{-1}$ $(n=21)$ and $9 \pm 13$ pA.pF $F^{-1}(n=8)$, respectively, while HEK293 clones transfected with hClCal express chloride currents at 5 to 10 times higher current density. Density of chloride currents recorded in hClCal clones 4 and 8 were $81 \pm 46$ pA.pF ${ }^{-1}(n=13)$ and 117 pA.pF $F^{-1} \pm 66$ $(n=14)$, respectively. Figure $6 G$ shows that untransfected (or wild type) NCIH522, pcDNA3.1 transfected NCIH522 and non-hClCa1 transfected NCIH522 all express a similar low density of chloride currents of about $5 \mathrm{pA} . \mathrm{pF}^{-1}$ whereas a 5 times higher density of chloride currents of $26 \pm 15$ pA.pF ${ }^{-1}$ can be observed in NCIH522 transfected with hClCal.

\section{$\mathrm{hClCa} 1$ affects the relative conductance between thiocyanate and chloride}

To gain an insight into the anion selectivity of hClCa1 extracellular chloride, $\mathrm{Cl}^{-}$was replaced with various anions (bi-ionic condition, Fig. 7), using thiocyanate $\left(\mathrm{SCN}^{-}\right)$, iodide $\left(\mathrm{I}^{-}\right)$, bromide $\left(\mathrm{Br}^{-}\right)$, isethionate and gluconate $\left(\mathrm{Gluc}^{-}\right)$. In Fig. $7 A$ and $B$, a ramp pulse protocol was applied in the presence of chloride and thiocyanate and the reversal potential of the currents was calculated to determine the relative permeability of the tested anions (Fig. $7 C-E$ ). Replacing external $\mathrm{Cl}^{-}$with $\mathrm{SCN}^{-}$, $\mathrm{I}^{-}, \mathrm{Br}^{-}$, isethionate and Gluc ${ }^{-}$induced a shift of the reversal potential which led to a permeability sequence of $\quad P_{\mathrm{SCN}^{-}}>P_{\mathrm{Cl}^{-}}{ }^{-}>{\mathrm{Br}^{-}}^{-}={P_{\mathrm{I}}}^{-}>P_{\text {isethionate }} \geq P_{\mathrm{Gluc}}{ }^{-}$. This was the case whether HEK293 or NCIH522 cells were transfected or untransfected with hClCal (Fig. 7C-E, left). Anionic conductance was then estimated from the chord conductance between $+80 \mathrm{mV}$ and the reversal potential (Fig. $7 C-E$, right) leading to an anionic conductance sequence of $G_{\mathrm{SCN}}{ }^{-}>G_{\mathrm{Cl}^{-}}{ }^{-}=G_{\mathrm{I}}{ }^{-}=G_{\mathrm{Br}}{ }^{-}>G_{\text {isethionate }}>G_{\mathrm{Gluc}}{ }^{-}$in hClCa1 untransfected cells. When HEK293 cells were stably transfected with hClCal, the conductance carried by the $\mathrm{Cl}^{-}$ion was increased from $1.4 \pm 1.2 \mathrm{nS}(n=11)$ and $1.5 \pm 1.47 \mathrm{nS}(n=10)$ in HEK293 wild type and HEK293-PCIN5, respectively, to $3.4 \pm 2.2 \mathrm{nS}(n=8)$ and $4.5 \pm 3.3 \mathrm{nS}(n=12)$ for HEK293-PCIN5-hClCal clone 4 and HEK293-PCIN5-hClCal clone 8, respectively.
This is in accordance with an increased chloride current in the presence of hClCal, as observed in Fig. 4 or in Fig. 6. The main effect of hClCal is described in Fig. $7 B$, showing similar current amplitudes in the presence of $\mathrm{SCN}^{-}$or $\mathrm{Cl}^{-}$, leading to $\mathrm{G}_{\mathrm{Cl}}{ }^{-}$ that equals $G_{\mathrm{SCN}^{-}}{ }^{-}$and to a conductance sequence of $G_{\mathrm{SCN}}{ }^{-}=G_{\mathrm{Cl}}{ }^{-}=G_{\mathrm{I}}{ }^{-}={G_{\mathrm{Br}}}^{-}>G_{\text {isethionate }}>G_{\mathrm{Gluc}}{ }^{-}$ (Fig. $7 C-E$ right).

\section{hClCa1 affects chloride channel energy barriers}

The increase in chloride current magnitude observed with hClCal expression could result from an increase

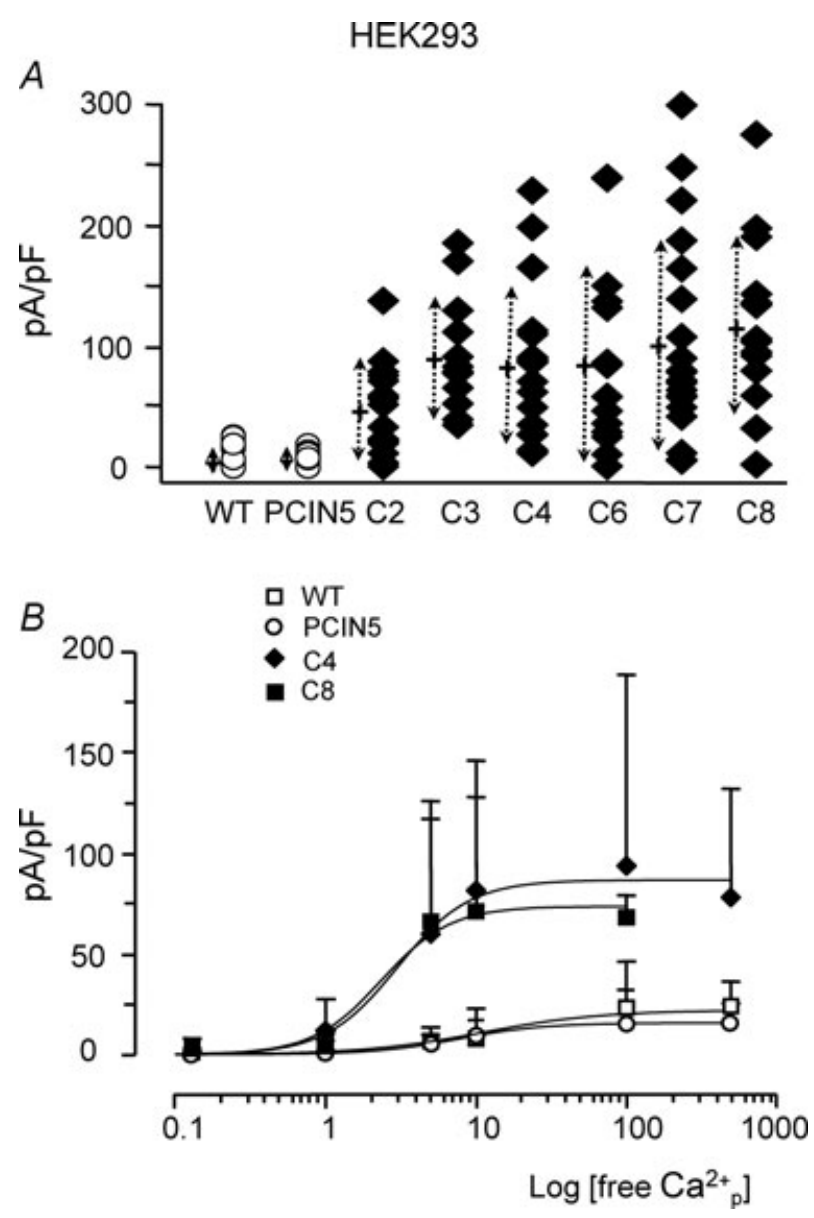

Figure 5. Density and calcium dependency of chloride currents in HEK293 cells

$A$, density of chloride currents recorded at a potential of $+80 \mathrm{mV}$ in HEK293 wild type (WT) and in HEK293 stably transfected with PCIN5 or with $\mathrm{pCIN5}-\mathrm{hClCa} 1$ clones 2, 3, and 4-8. The internal free calcium concentration was set at $10 \mu \mathrm{M}$. Symbols represent individual values obtained for each cells. Crosses and dotted arrows represent the mean and the standard deviation, respectively, besides each condition. $B$, density of chloride currents recorded at $+80 \mathrm{mV}$ in function of varying internal free calcium concentration (in the pipette) in HEK293 wild type (WT, $\square$ ), in HEK293 cells stably transfected with PCIN5 (O) or with pCIN5-hCICa1 clone $4(\boldsymbol{)})$ and clone $8(\boldsymbol{\square})$. Values were fitted according to a non-linear logistic function (see Methods). 
$\mathrm{NCl}-\mathrm{H} 522$

$A$

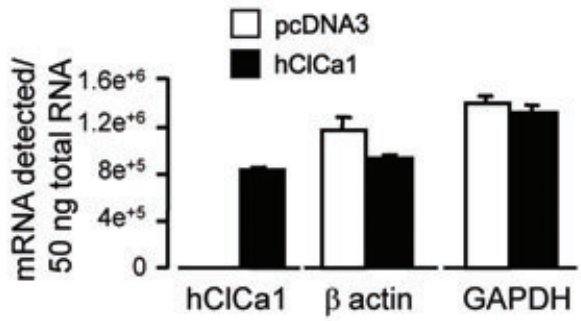

C

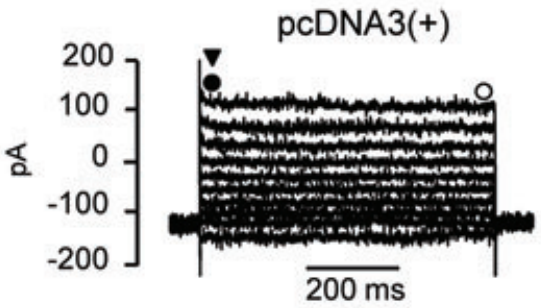

E

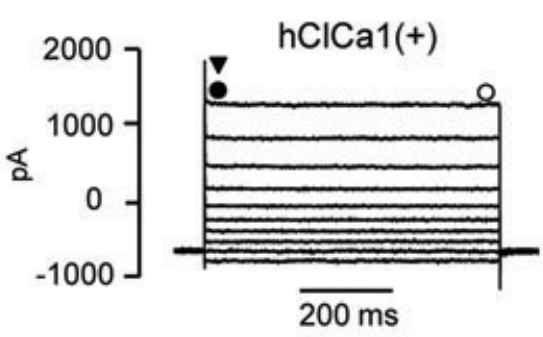

G

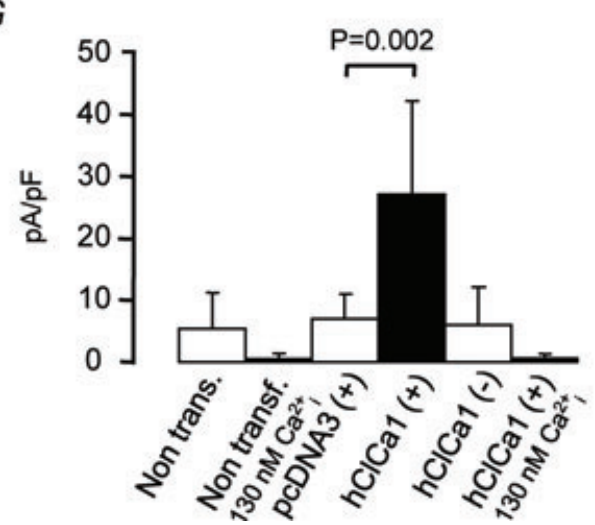

$B$

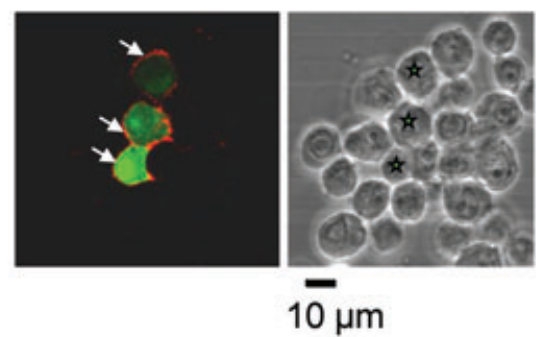

$D$

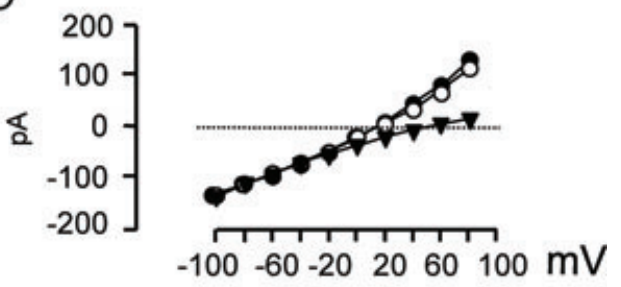

$F$

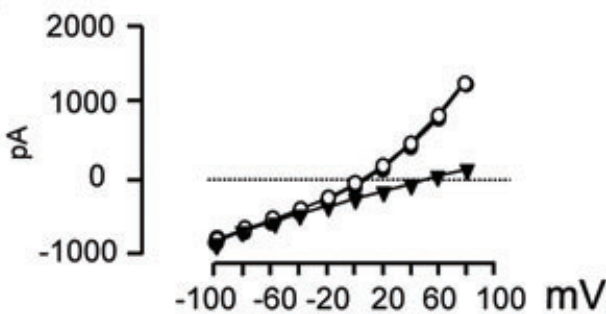

$H$

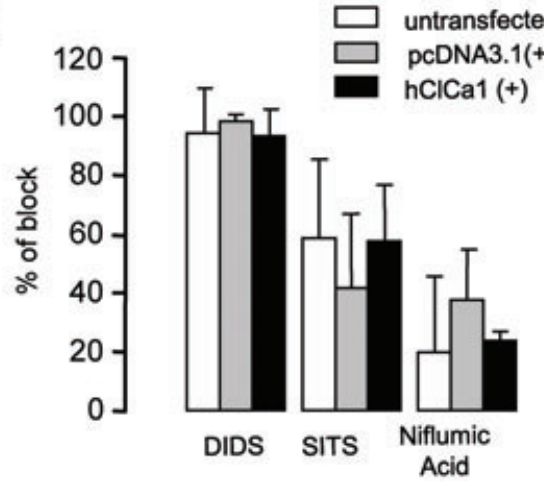

Figure 6. Characterisation of calcium-dependent chloride currents recorded from NCIH522 cells transiently transfected with $\mathrm{hClCa} 1$

$A$, Taqman quantitative PCR analysis of $\mathrm{hClCa1}, \beta$ actin and GAPDH, performed after mRNA extraction from $\mathrm{NCIH522}$ cells transienly transfected with the vector pCDNA3 (open bars) or with pCDNA3-hClCa1 (filled bars). $B$, immunofluorescence light microscopy showing that A48 is labelling the surface of cells (arrows pointing to the labelling at the level of the membrane) transfected with green fluorescent protein-pcDNA3-hCICa1 (cytoplasmic labelling). Co-labelling is always observed (stars in right panel). C, representative whole cell currents from a $\mathrm{NCIH522}$ cell transiently transfected with pcDNA3, recorded during voltage steps from $-100 \mathrm{mV}$ to $+80 \mathrm{mV}$ (holding potential of $-80 \mathrm{mV}, 20 \mathrm{mV}$ step). $D$ represents the $I-V$ relationship of the currents measured in $C$ at the beginning of the step $(\bullet)$, at the end of the step (O) and after substituting chloride with gluconate $(\boldsymbol{\nabla})$. $E$, representative whole cell currents from a NCIH522 cell transiently transfected with pcDNA3-hClCa1, recorded in the same conditions as in $C$. $F$ represents the $I-V$ relationship of the currents measured in $E$ at the beginning of the step (•), at the end of the step (O) and after substituting chloride with gluconate $(\mathbf{v})$. Note that the circles are superimposed. Note the difference in the amplitude scale bar between $C$ and $E$ and between $D$ and F. G, summary histograms representing the density of chloride currents recorded at $+80 \mathrm{mV}$ in different conditions. 
in the number of open channels $\left(N P_{\text {open }}\right)$, an increase in single channel amplitude (i) or conductance $(g)$ or a combination of both. A decrease of the whole cell conductance ratio $G_{\mathrm{SCN}^{-}} / G_{\mathrm{Cl}^{-}}$suggests that hClCa1 expression affects the relative single channel conductance ratio $g_{\mathrm{SCN}^{-}} / g_{\mathrm{Cl}^{-}}$. To gain insight into possible changes in the pore induced by hClCal, we modelled the pore with a single binding site (see Methods for details) and obtained fits to whole cell $I-V$ curves obtained with $126 \mathrm{~mm}$ of either $\mathrm{Cl}^{-}$or $\mathrm{SCN}^{-}$in the external solution and $130 \mathrm{~mm}$ $\mathrm{Cl}^{-}$in the pipette (Fig. 8). Energy profiles for both $\mathrm{Cl}^{-}$and $\mathrm{SCN}^{-}$were fitted simultaneously using the solver function in Microsoft Excel to minimize the sum of the squared difference between data and fit. During this procedure the values of the energy levels $G 12, G 2$ and $G 23$ for $\mathrm{Cl}^{-}$ and $\mathrm{SCN}^{-}$were free to alter as was the number of open channels $\left(N P_{\text {open }}\right)$. As seen in Fig. 8 , this single site model fitted the data adequately in the presence or absence of hClCa1 expression in either HEK 293 or NCIH522 cells. It was clear, however, that to obtain an adequate fit to the data it was necessary to alter the energy barriers, as simply scaling the current by altering the number of open channels depending on the presence or absence of hClCal was inadequate (see dashed lines in Fig. 8). The single channel conductance (estimated as the average slope conductance) in $126 \mathrm{mM} \mathrm{Cl}^{-}$was $3.7 \mathrm{pS}$ in the HEK 293 stable cell line and this increased to $11.2 \mathrm{pS}$ in the presence of hClCa1 (Table 3). Similar results were obtained with transient transfection using pcDNA3.1 (Table 4). This increased conductance in the presence of hClCal was mainly reflected by a decrease in the value of $G 12$, the external energy barrier, for $\mathrm{Cl}^{-}$, which also accounted for the change in the conductance ratio $\left(G_{\mathrm{SCN}^{-}} / G_{\mathrm{Cl}^{-}}\right)$ observed. Even though $N P_{\text {open }}$ was free to change during the fitting routine, the value returned was remarkably consistent irrespective of the presence of hClCal.

\section{Discussion}

In the present study, RT-PCR techniques and immunocytochemistry labelling using the specific A48 antibody
(Gibson et al.2005) were used to investigate the expression of hClCa1 in HEK293 and NCIH522, cell types that have not been shown to express $\mathrm{ClCa}$ isoforms. We proceeded to a functional characterisation of hClCal in HEK293 stable cell lines expressing hClCal as well as in HEK293 and NCIH522 cells transiently expressing hClCal, and accumulated evidence that hClCal does not constitute an independent channel but is a $\mathrm{Ca}^{2+}$-dependent chloride channel modulator, lowering energy barriers of the channel.

\section{Properties of the calcium-dependent chloride currents in HEK293 and NCIH522}

Species-dependent isoforms of $\mathrm{ClCa}$ gene products have been described in HEK293 cells (Gandhi et al. 1998; Gruber et al. 1998, 1999). In all those cases, the expressed current reversed close to $0 \mathrm{mV}$, exhibited some outward rectification but no time-dependent kinetics. Similarly, we showed that $\mathrm{Ca}^{2+}$-dependent chloride currents upregulated in HEK293 and NCIH522 cells reversed close to $0 \mathrm{mV}$, exhibited some outward rectification and mainly time-independent kinetics. On step-like depolarisations, the instantaneous current was not followed by an outward relaxation similar to other studies performed on HEK293 cells (Gandhi et al. 1998; Gruber et al. 1998, 1999; Greenwood et al. 2002) and this is in contrast to various cell types including smooth muscle, secretory and epithelial cells chloride currents that exhibit distinctive voltageand time-dependent characteristics (Large \& Wang, 1996; Frings et al. 2000). It is possible that auxiliary subunits are required to exhibit distinctive voltage-dependent kinetics characteristic of native $\mathrm{Ca}^{2+}$-dependent chloride currents as suggested for the $\mathrm{BK} \beta$ subunit that can associate and modulate mClCA1 to get a $\mathrm{Ca}^{2+}$ sensitivity more typical of the native currents in smooth muscle cells (Greenwood et al. 2002). In previous studies on HEK293 cells (Gandhi et al. 1998; Gruber et al. 1998, 1999), $\mathrm{Ca}^{2+}$-dependent chloride currents required a high concentration of $\mathrm{Ca}^{2+}(2 \mathrm{mM})$ for activation. In our experimental conditions, $\mathrm{Ca}^{2+}$-dependent chloride

The first column represents the density of chloride currents in wild type NCIH522 cells (internal free calcium concentration of $10 \mu \mathrm{M}$ ). The second column represents the current density in wild type NCIH522 cells recorded with an internal free calcium concentration of $130 \mathrm{~nm}$. Note the presence of a small background current when cells are recorded in the presence of $10 \mu \mathrm{m}$ internal free calcium concentration. Columns 3 and 4 represent currents obtained in NCIH522 cells transfected with the vector pCDNA3 and with pcDNA3-hClCa1, respectively (both conditions with internal free calcium concentration of $10 \mu \mathrm{M}$ ). Note that the presence of hClCa1 increases the density of the chloride current. Column 5 represents the density of the chloride current in NCIH522 cells that were incubated with pcDNA3-hClCa1 but stayed untransfected (unlabelled, recorded internal free calcium concentration of $10 \mu \mathrm{M})$. Column 6 represents the density of the chloride current obtained in NCIH522 cells transfected with pcDNA3-hClCa1 and recorded at an internal free calcium concentration of $130 \mathrm{~nm}$. $\mathrm{H}$, percentage of block of the chloride current by $300 \mu \mathrm{M}$ DIDS, $300 \mu \mathrm{M}$ SITS and $100 \mu \mathrm{M}$ niflumic acic. Current was recorded at $+80 \mathrm{mV}$ from a holding potential of $-80 \mathrm{mV}$ in untransfected NCIH522 cells (open bars), NCIH522 transfected with pcDNA3.1 and NCIH522 transfected with pCDNA3.1-hClCa1. 
currents upregulated by hClCal were activated by a lower concentration of $\mathrm{Ca}^{2+}$ (over $150 \mathrm{nM}$ ) which is close to the $100 \mathrm{~nm}$ threshold of activation of outwardly rectifying $\mathrm{Ca}^{2+}$-dependent chloride currents in lacrimal acinar cells (Evans \& Marty, 1986) or in various cell types including smooth muscles (Large \& Wang, 1996; Wang et al. 1997), cardiac myocytes (Collier et al. 1996) or endothelial cells (Nilius et al. 1997) and is also near the resting level of $\mathrm{Ca}^{2+}$ in secretory epithelia (Kidd \& Thorn, 2000). $\mathrm{Ca}^{2+}$-dependent chloride currents showed
A

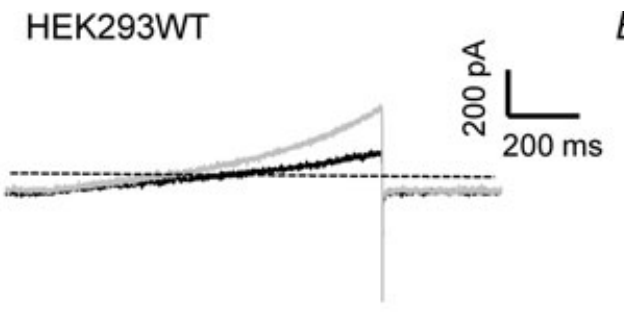

B $\quad \mathrm{HEK} 293 \mathrm{hClCa} 1$

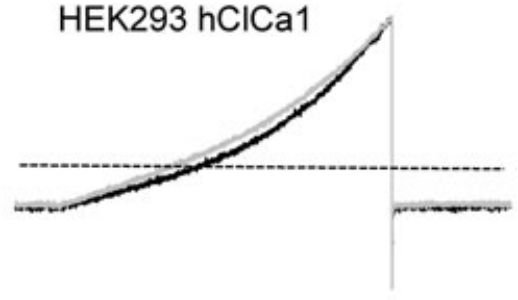

C

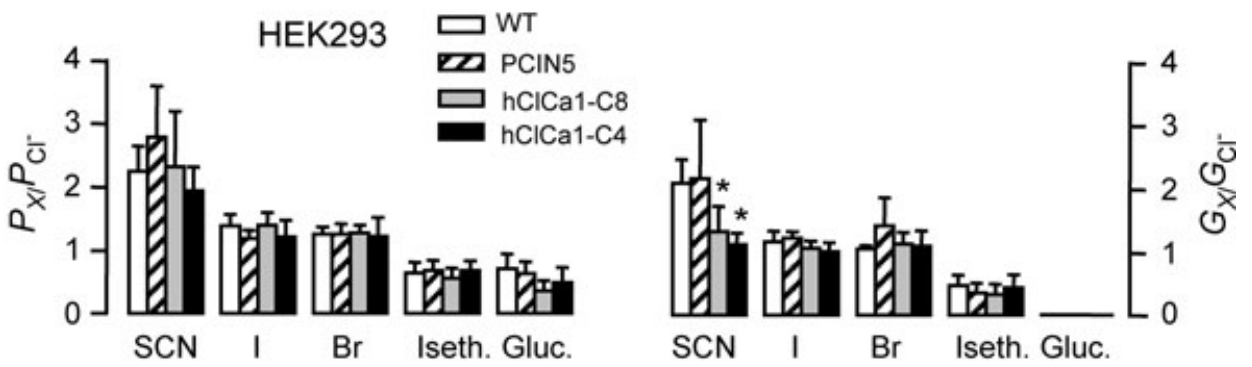

$D$

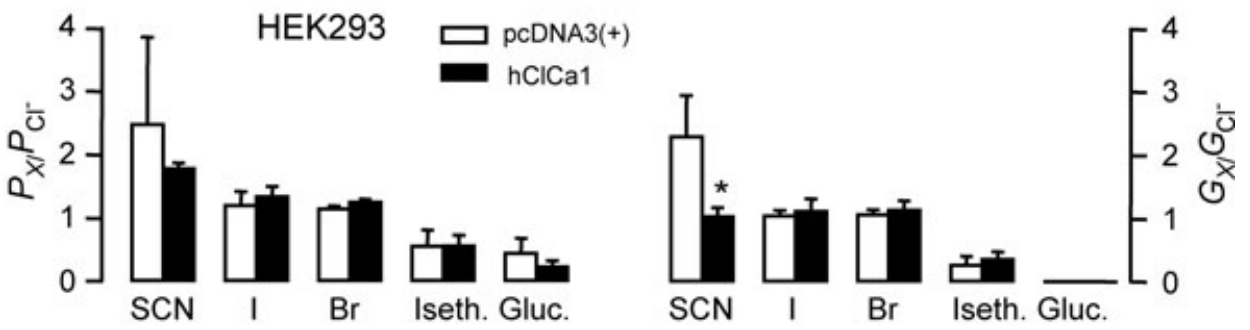

E
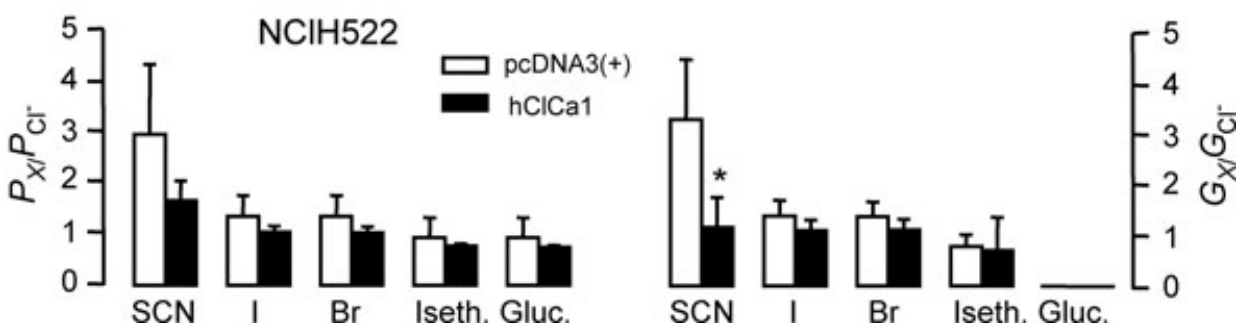

Figure 7. Relative anionic conductance and permeability in HEK293 and $\mathrm{NCIH} 522$ cells

A, representative currents recorded in HEK293 wild type cells during a voltage ramp protocol from a holding potential of $-80 \mathrm{mV}$ to $+100 \mathrm{mV}$ in control condition (black) and after substituting chloride with thiocyanate (grey). $B$, representative currents recorded in the same conditions in HEK293 cells stably transfected with PCIN5-hCICa1. Dashed lines in both $A$ and $B$ represent the null value of the current. $C-E$, summary histograms of the anionic permeability ratios (left) and the conductance ratios (right) obtained in the different cell types (see below). The relative anionic permeability is calculated from the shift of the reversal potential measured after substituting chloride with thiocyanate (SCN), iodide (I), bromide (Br), isethionate (Iseth) and gluconate (Gluc). The conductance was determined by dividing the measured current at $+80 \mathrm{mV}$ by the difference between the membrane potential and the membrane at which there is zero current. The relative conductance is the ratio of the conductance values obtained in different anionic conditions. $C$ is a summary of the anionic selectivity measured in HEK293 wild type, stably transfected with PCIN5 or stably transfected with PCIN5-hClCa1 (clones 4 and 8). D is a summary of the anionic selectivity measured in the same condition as described above in HEK293 cells transiently transfected with pcDNA3 or in HEK293 transiently transfected PCDNA3-hClCa1 (hClCa1). $E$ is a summary of the anionic selectivity measured in the same condition as described above in NCIH522 cells transiently transfected with pcDNA3 or in $\mathrm{NClH} 522$ transiently transfected PCDNA3-hClCa1 (hClCa1). 
a non-linear dependence of the current relative to the free $\mathrm{Ca}^{2+}$ concentration suggesting that the mechanisms of calcium-dependent chloride channel activation could involve the binding of multiple $\mathrm{Ca}^{2+}$ ions to the channels or to unknown accessory proteins. In lacrimal acinar cells, the $\mathrm{EC}_{50}$ of $\mathrm{Ca}^{2+}$ dependence was $\sim 1 \mu \mathrm{M}$ (Evans \& Marty, 1986) and our $\mathrm{EC}_{50}$ values in the micromolar range are also in agreement with this figure. In our experiments, the $\mathrm{Ca}^{2+}$ concentration was maintained at $10 \mu \mathrm{M}$ which is $\sim 2-3$ times the $K_{\mathrm{d}}$ of the channel for $\mathrm{Ca}^{2+}$ in Xenopus oocytes (Kuruma \& Hartzell, 2000).

\section{Effect of $\mathrm{Cl}^{-}$channel blockers}

Ion channel blockers have been used extensively as tools for the identification of $\mathrm{Ca}^{2+}$-dependent chloride currents. Unfortunately, most of the known $\mathrm{Cl}^{-}$channel blockers are neither selective nor potent (Greenwood \&
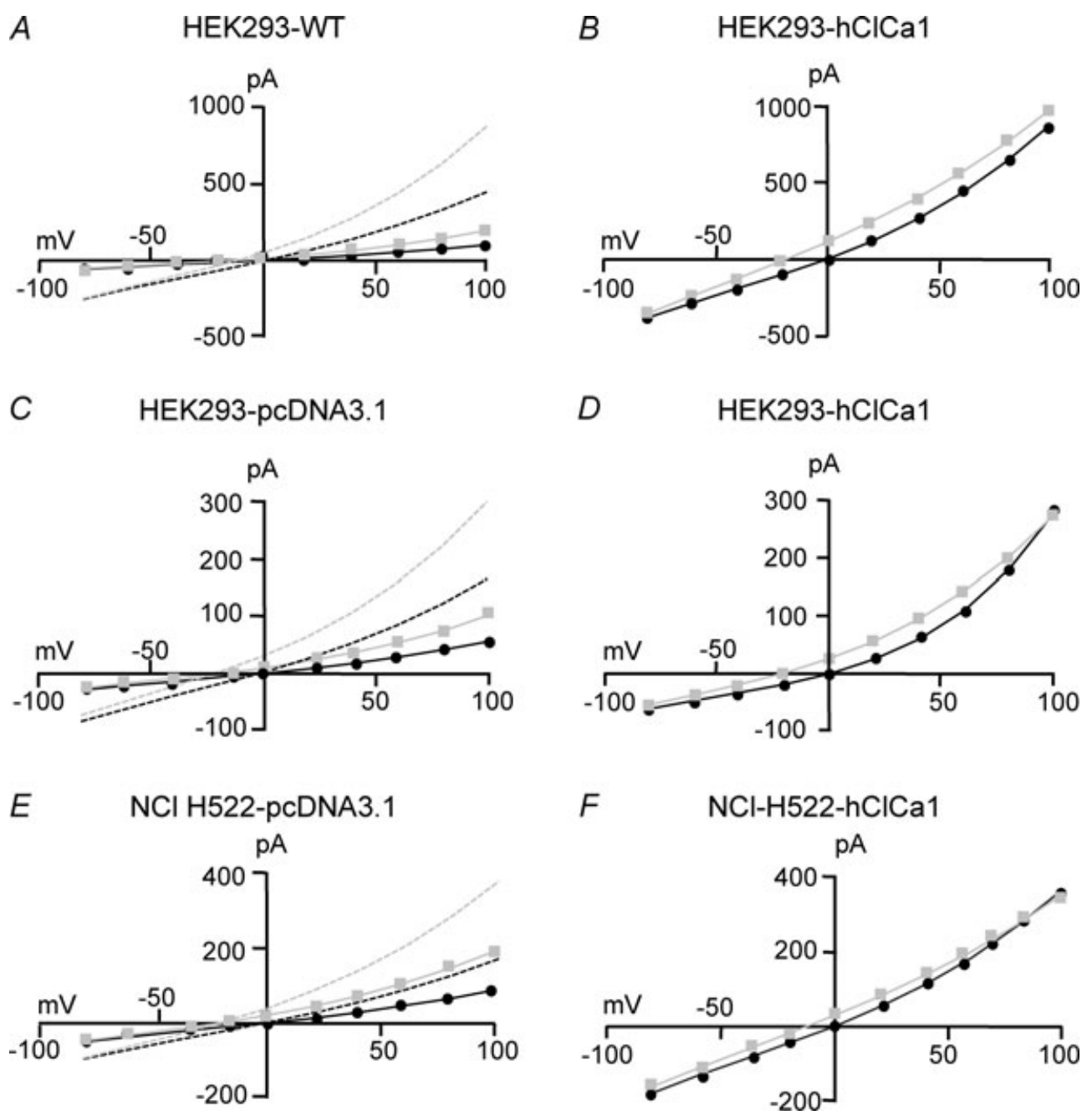

Figure 8. Current-voltage relationships fitted with a one energy well and two barriers model

$A-E$, typical current-voltage relationships recorded from the different cell types in presence of $135 \mathrm{~mm}$ external $\mathrm{Cl}^{-}$(black circles) or $135 \mathrm{~mm} \mathrm{SCN}^{-}$(grey squares). Cell types are mentioned above each $\mathrm{I} V$ curves. Continuous lines represent the fit through the data with the values of the energy levels $\mathrm{G} 12, \mathrm{G} 2$ and $\mathrm{G}_{2} 3$ for $\mathrm{Cl}^{-}$and SCN${ }^{-}$ free to alter and the number of open channels free to alter. Dashed lines in $A, C$ and $E$ represent the same fit by scaling the continuous line current and multiplying the number of open channels by a factor of 2 to 4 . Individual values obtained were for: $A$ : $N=242, g=3.5 \mathrm{pS}$; with $\mathrm{Cl}^{-}: \mathrm{G} 12$ 6.5 RT, G2 $-4 R T, G 23$ 6.9; with SCN $\mathrm{SCN}^{-}$: G12 5.6RT, G2 -6.0 RT, G23 6.6. Dashed line represents the fit with $N \times 4$ fixed; for $B: N=231, g=30 \mathrm{pS}$; with $\mathrm{Cl}^{-}$: G12 4.1 RT, G2 -5.8RT, G23 5.0 RT; with SCN-: G12 4.3RT, G2 -5.0 RT, G23 3.7 RT; for C: $N=263$, $g=1.8 \mathrm{pS}$; with $\mathrm{Cl}^{-}$: G12 5.7 RT, G2 -10.5 RT, G23 5.0 RT; with SCN': G12 5.3 RT, G2 -11.9.0 RT, G23 3.2 RT. Dashed line represents the fit with $N \times 3$ fixed; for $D: N=239, g=10.5 \mathrm{pS}$; with $\mathrm{Cl}^{-}: \mathrm{G} 124.7 R T, G 2-5.8 R T$, G23 6.9 RT; with SCN ${ }^{-}$: G12 3.8RT, G2 -8.2 RT, G23 6.1 RT; for $E: N=242, g=3.1 \mathrm{pS}$; with $\mathrm{Cl}^{-}: \mathrm{G} 126.3 R T$, G2 -7.5 RT, G23 7.0 RT; with SCN ${ }^{-}$: G12 5.9 RT, G2 -6.0 RT, G23 6.0 RT. Dashed line represents the fit with $N \times 2$ fixed; for $F: N=236, g=12.7 \mathrm{pS}$; with $\mathrm{Cl}^{-}: \mathrm{G} 125.2 R T, G 2-4.7 R T, G 235.8 R T$; with $\mathrm{SCN}^{-}: \mathrm{G} 125.1 R T$, G2 -8.0 RT, G23 5.1 RT. 
Table 3. Energy barriers estimated from a single energy barrier model in the presence and absence of hClCa1 in HEK293 stable cell lines

\begin{tabular}{llccc}
\hline & & HEK293-WT & HEK293-hClCa1 & \\
& & HEK-PCIN5 & C4,C8 & $P$ \\
\hline $\mathrm{Cl}^{-}$ & G12 & $6.7 \pm 0.7$ & $5.34 \pm 0.4$ & 0.002 \\
& G2 & $-7.8 \pm 3.4$ & $-5.3 \pm 4.7$ & 0.31 \\
& G23 & $2.1 \pm 2.3$ & $3.7 \pm 2.9$ & 0.32 \\
$\mathrm{SCN}^{-}$ & $G 12$ & $4.3 \pm 1.8$ & $2.6 \pm 1.9$ & 0.15 \\
& $G 23$ & $-9.4 \pm 2.0$ & $-8.1 \pm 1.9$ & 0.48 \\
& $G 3$ & $5.2 \pm 2.3$ & $4.6 \pm 1.6$ & 0.58 \\
& $N$ & $398 \pm 14.1$ & $389 \pm 11.6$ & 0.24 \\
& $G(p S)$ & $3.7 \pm 1.9$ & $11.2 \pm 4.8$ & 0.0003 \\
\hline
\end{tabular}

Data are mean \pm S.D. $(n=6)$ for each column. Left column comprises the data obtained in HEK293-WT and HEK293 stably transfected with PCIN5 whereas the column on the right shows values obtained from HEK293 stably transfected with PCIN5-hClCa1 (clones 4 and 8). $G$ are in RT units whereas $N$ represents the total numbers of channels $\times$ opening probability. $g$ represents the conductance in pS. $P$ values are obtained from a Student's $t$ test.

Table 4. Energy barriers estimated from a single energy barrier model in the presence and absence of $\mathrm{hClCa} 1$ in transiently transfected HEK293 and NCIH522

\begin{tabular}{|c|c|c|c|c|}
\hline & & $\begin{array}{l}\text { HEK293, } \\
\text { NCIH522- } \\
\text { pcDNA3.1 }\end{array}$ & $\begin{array}{c}\text { HEK293, } \\
\text { NCIH522-hClCa1 } \\
\text { C4, C8 }\end{array}$ & $P$ \\
\hline \multirow[t]{3}{*}{$\mathrm{Cl}^{-}$} & G12 & $6.2 \pm 0.8$ & 4.8. \pm 1.0 & 0.01 \\
\hline & G2 & $-8.4 \pm 2.6$ & $-4.8 \pm 3.0$ & 0.04 \\
\hline & G23 & $4.2 \pm 3.4$ & $5.9 \pm 0.7$ & 0.21 \\
\hline \multirow[t]{5}{*}{$\mathrm{SCN}^{-}$} & G12 & $4.6 \pm 2.3$ & $3.7 \pm 1.4$ & 0.38 \\
\hline & G23 & $-6.7 \pm 4.9$ & $-6.8 \pm 2.3$ & 0.94 \\
\hline & G3 & $5.0 \pm 1.9$ & $5.3 \pm 1.0$ & 0.7 \\
\hline & $N$ & $245 \pm 14.4$ & $237 \pm 5.0$ & 0.24 \\
\hline & $g(p S)$ & $2.8 \pm 1.3$ & $10.0 \pm 2.3$ & 0.0001 \\
\hline
\end{tabular}

Data are mean \pm S.D. $(n=6)$ for each column. Left column comprises the data obtained in HEK293 and NCIH522 transiently with pcDNA3.1(+) whereas the column on the right shows values obtained from HEK293 and NCIH522 transiently transfected with pcDNA3.1(+)-hClCa1 (clones 4 and 8). $G$ are in RT units whereas $N$ represents the total numbers of channels $\times$ opening probability. $g$ represents the conductance in pS. $P$ values are obtained from a Student's $t$ test.

Leblanc, 2007). Commonly used stillbene disulphonate and niflumic acid display inhibitory effects on various cell types (Greenwood \& Leblanc, 2007) and we found that, similar to previous reports (Gandhi et al. 1998; Gruber et al. 1998), DIDS blocked the current by about $90 \%$ in the absence and presence of hClCal. DIDS also exerted a more potent block at positive potentials consistent with the voltage dependency of the blocking effect reported in previous studies (Nilius et al. 1997; Kim et al. 2003). Niflumic acid (100 $\mu \mathrm{M})$ was a relatively weak blocker in HEK293 and NCIH522 cells even at positive potentials. This differs from previous studies (Gandhi et al. 1998; Gruber et al. 1998) showing that niflumic acid abolished the calcium-dependent chloride current in HEK293 cells. One reason for this difference could be the intracellular calcium concentration. Whereas we used $10 \mu \mathrm{M} \mathrm{Ca}^{2+}$ concentration, previous studies used $2 \mathrm{mM}$ $\mathrm{Ca}^{2+}$ inside the recording pipette. We did not observe complicated effects of niflumic acid like those reported in vascular smooth muscle where niflumic acid inhibits the calcium-dependent chloride current at positive potentials whilst increasing it at negative potentials (Piper et al. 2002).

\section{hClCa1 is a $\mathrm{Ca}^{2+}$-dependent chloride current modulator}

hClCal has originally been suggested to be an ion channel of the calcium-dependent chloride channel family (Gandhi et al. 1998; Gruber et al. 1998). Later observations showed that hClCa1, as well as cleavage products of $\mathrm{mClCa} 3$, the murine homolog of hClCa1, are non-integral membrane proteins released into the medium. In their studies, the authors conclude that hClCal cannot form an anion channel on its own (Gibson et al. 2005; Mundhenk et al. 2006). Furthermore, bioinformatics analyses do not predict the presence of any transmembrane domains and thus hClCal is unlikely to be an ion channel per se. In their initial study, Gruber et al. (1998) have shown that transient expression of hClCa1 in HEK293 cells increased the $\mathrm{Ca}^{2+}$-activated whole cell currents by a factor of 5, producing a time-independent outwardly rectifying current. As there was no mention of endogenous HEK293 channels that could be responsible for a background current mediated by non-selective anion currents, the conclusion was drawn towards hClCal being an ion channel itself. By opposition to Gruber et al. (1998), we have shown that both HEK 293 and NCIH522 cell lines express, in the absence of hClCla1, a small (i.e. about $5 \mathrm{pA} . \mathrm{pF}^{-1}$ ) calcium-dependent chloride current with $10 \mu \mathrm{M}$ free internal calcium. In both cell lines, $\mathrm{Ca}^{2+}$-dependent $\mathrm{Cl}^{-}$currents were outwardly rectifying, time independent and inhibited by DIDS, SITS and niflumic acid, similarly to currents described by Gruber et al. 1998 when hClCa1 was expressed in HEK293 cells.

Our study shows that the expression of hClCal upregulates similar types of calcium-dependent chloride channel activity in transiently transfected HEK 293, NCIH522 cells or in clonal cultures of HEK293 cells expressing constitutively $\mathrm{hClCa}$ thereby confirming the observation of Gruber et al. (1998)) that the expression 
of hClCal greatly increased time-independent, outwardly rectifying $\mathrm{Ca}^{2+}$-dependent $\mathrm{Cl}^{-}$currents. Differences in the amplitudes of the $\mathrm{Ca}^{2+}$-activated $\mathrm{Cl}^{-}$currents are highly significant (i.e. by a factor of 10 to 20, leading to currents in the nanoamp range) when hClCal is expressed. The presence of $\mathrm{Ca}^{2+}$-activated $\mathrm{Cl}^{-}$channels prior to transfection suggests that $\mathrm{hClCal}$ is not encoding for novel $\mathrm{Ca}^{2+}$-dependent $\mathrm{Cl}^{-}$channels but rather modulates the amplitude of pre-existing $\mathrm{Ca}^{2+}$-dependent $\mathrm{Cl}^{-}$channels of similar biophysical and pharmacological properties. CLC-3 and bestrophin gene expression also cause the appearance of $\mathrm{Ca}^{2+}$-dependent $\mathrm{Cl}^{-}$currents that lack the typical voltage dependence of $\mathrm{Ca}^{2+}$-dependent $\mathrm{Cl}^{-}$currents seen, for example, in the parotid gland (Arreola et al. 1996) and the possibility exists that hClCa1 is involved in the up-regulation of such channels. Nevertheless, bestrophin or CLC-3 properties do not conform to the behaviour of the $\mathrm{Ca}^{2+}$-dependent $\mathrm{Cl}^{-}$ current observed in our study. $\mathrm{Ca}^{2+}$ concentrations higher than $1 \mu \mathrm{M}$ have an inhibitory effect on $\mathrm{Ca}^{2+}$-dependent $\mathrm{Cl}^{-}$currents expressed by bestrophins (Barro-Soria et al. 2008) and ClC-3 expression induced currents with time-dependent activation that rectified sharply in the outward direction (Matsuda et al. 2008).

We checked whether an increase in the affinity for internal calcium would account for the effect of $\mathrm{hClCa}$ on the amplitude of the current by measuring the amplitude of the $\mathrm{Ca}^{2+}$-dependent $\mathrm{Cl}^{-}$current at different internal free $\mathrm{Ca}^{2+}$ concentrations ranging from $130 \mathrm{nM}$ to $486 \mu \mathrm{M}$. We showed that hClCal drastically increases the amplitude of the $\mathrm{Ca}^{2+}$-dependent $\mathrm{Cl}^{-}$currents including maximal current amplitudes obtained at $486 \mu \mathrm{M}$ internal free $\mathrm{Ca}^{2+}$ concentration. Therefore, the small decrease of the $\mathrm{EC}_{50}$ value in the presence of hClCal cannot account for the overall increase of the current amplitude.

Our observations show variability in the expression of hClCa1 mRNA, hClCa1 protein or the amplitude of the chloride currents. Discrepancies also exist between the different expression levels (mRNA, immunolabelling or chloride currents). For example, HEK293 clone 6 expresses low levels of hClCal mRNA or A48 immunolabelling but has chloride currents of similar amplitude to those recorded in clones 4 and 8 . In fact, the absence of a proportional relationship between the level of expression of hClCal and the up-regulation of the $\mathrm{Ca}^{2+}$-dependent $\mathrm{Cl}^{-}$current supports the idea that hClCal is not encoding per se for a $\mathrm{Ca}^{2+}$-dependent $\mathrm{Cl}^{-}$channel but is rather a modulator likely to require other proteins in order to get reproducible, quantifiable and predictive effects. Our study also confirms observations with $\mathrm{pClCa} 1$, the porcine ortholog of hClCal, that suggested that $\mathrm{ClCal}$ was a chloride channel regulator that increased the amplitude of endogenous $\mathrm{Ca}^{2+}$-dependent $\mathrm{Cl}^{-}$channels whose identity remains unknown (Loewen et al. 2002, 2003, 2004). Finally, our results are in concordance with the effects of $\mathrm{mClCa} 3$, the mouse homolog of $\mathrm{hClCa}$, increasing the amplitude of time-independent and outwardly rectifying $\mathrm{Ca}^{2+}$-dependent $\mathrm{Cl}^{-}$currents (Winpenny et al. 2002).

Therefore, hClCal appears to be acting as a chloride channel regulator, increasing the amplitude of endogenous $\mathrm{Ca}^{2+}$-dependent $\mathrm{Cl}^{-}$chloride channels whose identity remains unknown. Gibson et al. (2005) showed that $\mathrm{hClCa}$ can be secreted in the extracellular medium although it is possible that residual $\mathrm{hClCal}$ remains on the cell surface as shown on microvilli, allowing an interaction with the ion channel. Mouse ClCal interacts with the large conductance potassium channel $\beta$ subunit, increasing the calcium sensitivity of the BK current and evoking large $\mathrm{Ca}^{2+}$-dependent $\mathrm{Cl}^{-}$currents (Greenwood et al. 2002). Whereas in this latter study, a physical association between the two proteins could be demonstrated, resolution of the function of hClCal and of the regulatory characteristics will first require a greater understanding of the structure of the $\mathrm{Ca}^{2+}$-dependent $\mathrm{Cl}^{-}$channel(s) and of the pore-conducting subunit of the channel. The identification of proteins coding for the $\mathrm{Ca}^{2+}$-dependent $\mathrm{Cl}^{-}$current in HEK293 cells remains therefore to be elucidated alongside proteins interacting with hClCal. Recently, Caputo et al. (2008) identified TMEM16A as an intrinsic constituent of the calcium-dependent chloride channel and showed that specific mutagenesis of highly conserved amino acids changed intrinsic properties of the channel. Other family members, such as TMEM16F, TMEM16J or TMEM16K, may represent other types of $\mathrm{Cl}^{-}$channels with different biophysical properties and mechanisms of regulation and may ultimately be the channel modulated by hClCa1 .

\section{hClCa1 decreases $\mathrm{Ca}^{2+}$-dependent $\mathrm{Cl}^{-}$channel energy barriers}

Altogether our data suggest a modulatory effect of hClCa1 on existing $\mathrm{Ca}^{2+}$-dependent $\mathrm{Cl}^{-}$channels. We performed a substitution of $\mathrm{Cl}^{-}$with different anions and first analysed the permeability sequence (shift of the reversal potential) obtained in those different conditions. Whether in the presence or absence of hClCa1, the permeability sequence for all currents was similar to the permeability sequence reported for $\mathrm{Ca}^{2+}$-dependent $\mathrm{Cl}^{-}$channels in Xenopus oocytes and in other tissues (Qu \& Hartzell, 2000). We showed that hClCal leaves the shift of the reversal potential under bi-ionic conditions unaffected, reflecting a similar ability for anions to enter the pore (Qu \& Hartzell, 2000) in the absence or presence of hClCal.

Having shown that hClCa1 increased the amplitude of $\mathrm{Cl}^{-}$currents, we then checked whether whole current amplitudes (whole cell conductances, $G$ ) were similarly increased for all anions. We found that while hClCal increased both $G_{\mathrm{SCN}^{-}}$and $G_{\mathrm{Cl}^{-}}$, its relative effect on both 
conductances was different, leading to a proportional higher increase of the conductance when carried by $\mathrm{Cl}^{-}$ in comparison to $\mathrm{SCN}^{-}$. As a result, $\mathrm{hClCa}$ ldecreased the whole cell conductance ratio $G_{\mathrm{SCN}^{-}}{ }^{-} / \mathrm{G}_{\mathrm{Cl}}{ }^{-}$. This suggests that hClCal does not increase the ability of ions to enter the pore (permeability) but rather modifies their ability to traverse the entire length of the pore (conductance) (Qu \& Hartzell, 2000). Permeability ratios, determined by the difference between the energy required to dehydrate the anion and some stabilization energy due to the anion entering the pore, are not highly dependent on the channel structure. Likewise, permeability ratios turn out to be properties that are least affected by mutations generally. Therefore, the inability of hClCal to affect the permeability ratio suggests that hClCal does not modify the anionic dehydration and stabilisation energy while entering the pore. Mutations usually affect the conductance. For example, only minor conformational changes of the hydrophobic region of the pore are required to create macroscopic changes in conductance in the ACh receptor-channel (Corry, 2006).

To gain insight into the mechanisms underlying the effect of hClCal on the decreased whole cell conductance ratio $G_{\mathrm{SCN}^{-}}{ }^{-} / G_{\mathrm{Cl}}{ }^{-}$, we performed best fit energy profiles for $G_{\mathrm{SCN}^{-}}{ }^{-} / G_{\mathrm{Cl}}{ }^{-}$using a simple Eyring (Eyring et al. 1949) model. Best fit energy profiles estimated that hClCal is likely to neither encode for chloride channels nor alter its opening probability ( $N$ unaffected) but instead increases the $\mathrm{Cl}^{-}$channel conductance from an estimated $3 \mathrm{pS}$ to values exceeding $10 \mathrm{pS}$, mainly by lowering the first energy barrier $G 12$ and also the second energy barrier G2. Those estimated conductances are within the range of conductances previously reported for this channel. Calcium-dependent $\mathrm{Cl}^{-}$conductances as small as $2 \mathrm{pS}$ have been previously reported in Xenopus oocytes (Takahashi et al. 1987) and single channel conductances of $13 \mathrm{pS}$ have also been described in HEK293 cells after transfection with hClCal (Gruber et al. 1998). The modulatory effect of hClCal could originate from changes in the geometrical properties of the inner core of the channel and modulation of electrostatic interactions between the ion and polar groups within the channel, thereby affecting the free energy profile and lowering the channel energy barriers.

We do not exclude the possibility that $\mathrm{hClCa}$ induces other similar types of $\mathrm{Ca}^{2+}$-dependent $\mathrm{Cl}^{-}$channels not functionally expressed in the control cells but this possibility seems unlikely considering that the model only fits data when the total number of channels is unchanged. Newly expressed channels would then theoretically need to be accompanied by a proportional decrease of the number of channels already present in order for the model to fit the data. Our results also indicate that the biophysical and pharmacological properties of the $\mathrm{Ca}^{2+}$-dependent $\mathrm{Cl}^{-}$current are left unchanged after expressing hClCal.
Newly expressed channels would then theoretically display similar biophysical and pharmacological properties. Therefore, the most likely and simple explanation of the effect of hClCal lies in the increase of the conductance of the single channel.

We tried to verify the prediction from the modelling and measured single channels in cells expressing hClCal. Despite multiple attempts, those measurements were unsuccessful. This could be due to the small single channel conductance that makes the single channel current indistinguishable from the measurement noise. Another likely explanation is linked to the low probability of recording single channels. We estimated an average number of 250 channels in $15 \mathrm{pF}$ cells. Considering $1 \mathrm{pF}$ equals $1 \mu \mathrm{f} \mathrm{cm}^{-2}$, this would lead to the probability of measuring a channel in $1 \mu \mathrm{m}^{2}$ patches of 0.25 . Ion channels may also form clusters that are unequally distributed within the cell thereby decreasing the probability of success even further. Our modelling studies fitted adequately the effect of $\mathrm{hClCal}$ when increasing the single channel conductance and leaving the channel number unchanged. Similarities in channel sparseness and clustering in cells expressing hClCal can de facto explain why in the presence of hClCa1 we could not observe single channels.

\section{hClCa1: a target for asthma?}

Mucus gland hyperplasia and mucus overproduction and hypersecretion are main features of the airway during asthma. Chloride channels play a major role in the process of transepithelial electrolyte and fluid absorption or secretion and are also involved in the regulation of mucus overproduction (Anderson et al. 1992; Morris, 1999; Patel et al. 2008). Upregulation of $\mathrm{mClCa} 3$ in murine models of asthma is strongly associated with mucus overproduction (Nakanishi et al. 2001). In humans, Toda et al. (2002) have shown an up-regulation of hClCal in the IL-9-responsive mucus-producing epithelium of asthmatic subjects that supports the hypothesis that hClCal may be responsible for the overproduction of mucus in asthmatic subjects. Nakanishi et al. (2001) have highlighted the potential clinical advantage of hClCal inhibitors in animal models of asthma, thus hClCal inhibition in asthmatic patients may be a new therapeutic strategy. In summary, an upregulation of hClCal during an asthma attack suggests an alteration of $\mathrm{Ca}^{2+}$-dependent $\mathrm{Cl}^{-}$channel energy profiles that could be related to mucus overproduction.

\section{References}

Anderson MP, Sheppard DN, Berger HA \& Welsh MJ (1992). Chloride channels in the apical membrane of normal and cystic fibrosis airway and intestinal epithelia. Am J Physiol Lung Cell Mol Physiol 263, L1-L14. 
Arreola J, Melvin JE \& Begenisich T (1996). Activation of calcium-dependent chloride channels in rat parotid acinar cells. J Gen Physiol 108, 35-47.

Barro-Soria R, Schreiber R \& Kunzelmann K (2008). Bestrophin 1 and 2 are components of the $\mathrm{Ca}^{2+}$ activated $\mathrm{Cl}^{-}$conductance in mouse airways. Biochim Biophys Acta 1783, 1993-2000.

Britton FC, Ohya S, Horowitz B \& Greenwood IA (2002). Comparison of the properties of CLCA1 generated currents and $I_{\mathrm{Cl}(\mathrm{Ca})}$ in murine portal vein smooth muscle cells. J Physiol 539, 107-117.

Caputo A, Caci E, Ferrera L, Pedemonte N, Barsanti C, Sondo E, Pfeffer U, Ravazzolo R, Zegarra-Moran O \& Galietta LJ (2008). TMEM16A, a membrane protein associated with calcium-dependent chloride channel activity. Science 322, 590-594.

Collier ML, Levesque PC, Kenyon JL \& Hume JR (1996). Unitary $\mathrm{Cl}^{-}$channels activated by cytoplasmic $\mathrm{Ca}^{2+}$ in canine ventricular myocytes. Circ Res 78, 936-944.

Corry B (2006). An energy-efficient gating mechanism in the acetylcholine receptor channel suggested by molecular and Brownian dynamics. Biophys J 90, 799-810.

Davies NW, McKillen HC, Stanfield PR \& Standen NB (1996). A rate theory model for $\mathrm{Mg}^{2+}$ block of ATP-dependent potassium channels of rat skeletal muscle. J Physiol 490, 817-826.

Eggermont J (2004). Calcium-activated chloride channels: (un)known, (un)loved? Proc Am Thorac Soc 1, 22-27.

Evans MG \& Marty A (1986). Calcium-dependent chloride currents in isolated cells from rat lacrimal glands. J Physiol 378, 437-460.

Eyring H, Lumry R \& Woodbury JW (1949). Some applications of modern rate theory to physiological systems. Rec Chem Prog 100, 100-114.

Frings S, Reuter D \& Kleene SJ (2000). Neuronal $\mathrm{Ca}^{2+}$-activated $\mathrm{Cl}^{-}$channels-homing in on an elusive channel species. Prog Neurobiol 60, 247-289.

Gandhi R, Elble RC, Gruber AD, Schreur KD, Ji HL, Fuller CM \& Pauli BU (1998). Molecular and functional characterization of a calcium-sensitive chloride channel from mouse lung. J Biol Chem 273, 32096-32101.

Gibson A, Lewis AP, Affleck K, Aitken AJ, Meldrum E \& Thompson N (2005). hCLCA1 and mCLCA3 are secreted non-integral membrane proteins and therefore are not ion channels. J Biol Chem 280, 27205-27212.

Glässtone S, Laidler KJ \& Eyring H (1941). The Theory of Rate Processes: The Kinetics of Chemical Reactions, Viscosity, Diffusion and Electrochemical Phenomena. McGraw-Hill, New York.

Greenwood IA \& Leblanc N (2007). Overlapping pharmacology of $\mathrm{Ca}^{2+}$-activated $\mathrm{Cl}^{-}$and $\mathrm{K}^{+}$channels. Trends Pharmacol Sci 28, 1-5.

Greenwood IA, Miller LJ, Ohya S \& Horowitz B (2002). The large conductance potassium channel $\beta$-subunit can interact with and modulate the functional properties of a calcium-activated chloride channel, CLCA1. J Biol Chem 277, 22119-22122.
Gruber AD, Elble RC, Ji HL, Schreur KD, Fuller CM \& Pauli BU (1998). Genomic cloning, molecular characterization, and functional analysis of human CLCA1, the first human member of the family of $\mathrm{Ca}^{2+}$-activated $\mathrm{Cl}^{-}$channel proteins. Genomics 54, 200-214.

Gruber AD, Schreur KD, Ji HL, Fuller CM \& Pauli BU (1999). Molecular cloning and transmembrane structure of hCLCA2 from human lung, trachea, and mammary gland. Am J Physiol Cell Physiol 276, C1261-C1270.

Hartzell C, Putzier I \& Arreola J (2005). Calcium-activated chloride channels. Annu Rev Physiol 67, 719-758.

Hauber HP, Manoukian JJ, Nguyen LH, Sobol SE, Levitt RC, Holroyd KJ, McElvaney NG, Griffin S \& Hamid Q (2003). Increased expression of interleukin-9, interleukin-9 receptor, and the calcium-activated chloride channel hCLCA1 in the upper airways of patients with cystic fibrosis. Laryngoscope 113, 1037-1042.

Hauber HP, Tsicopoulos A, Wallaert B, Griffin S, McElvaney NG, Daigneault P, Mueller Z, Olivenstein R, Holroyd KJ, Levitt RC \& Hamid Q (2004). Expression of HCLCA1 in cystic fibrosis lungs is associated with mucus overproduction. Eur Respir J 23, 846-850.

Hoshino M, Morita S, Iwashita H, Sagiya Y, Nagi T, Nakanishi A, Ashida Y, Nishimura O, Fujisawa Y \& Fujino M (2002). Increased expression of the human $\mathrm{Ca}^{2+}$-activated $\mathrm{Cl}^{-}$ channel 1 (CaCC1) gene in the asthmatic airway. Am J Respir Crit Care Med 165, 1132-1136.

Jentsch TJ, Stein V, Weinreich F \& Zdebik AA (2002). Molecular structure and physiological function of chloride channels. Physiol Rev 82, 503-568.

Kidd JF \& Thorn P (2000). Intracellular $\mathrm{Ca}^{2+}$ and $\mathrm{Cl}^{-}$ channel activation in secretory cells. Annu Rev Physiol 62, 493-513.

Kim SJ, Shin SY, Lee JE, Kim JH \& Uhm SY (2003). Ca ${ }^{2+}$ activated $\mathrm{Cl}^{-}$channel currents in rat ventral prostate epithelial cells. Prostate 55, 118-127.

Kuruma A \& Hartzell HC (2000). Bimodal control of a $\mathrm{Ca}^{2+}$-activated $\mathrm{Cl}^{-}$channel by different $\mathrm{Ca}^{2+}$ signals. J Gen Physiol 115, 59-80.

Large WA \& Wang Q (1996). Characteristics and physiological role of the $\mathrm{Ca}^{2+}$-activated $\mathrm{Cl}^{-}$conductance in smooth muscle. Am J Physiol Cell Physiol 271, C435-C454.

Loewen ME, Bekar LK, Walz W, Forsyth GW \& Gabriel SE (2004). pCLCA1 lacks inherent chloride channel activity in an epithelial colon carcinoma cell line. Am J Physiol Gastrointest Liver Physiol 287, G33-G41.

Loewen ME \& Forsyth GW (2005). Structure and function of CLCA proteins. Physiol Rev 85, 1061-1092.

Loewen ME, Gabriel SE \& Forsyth GW (2002). The calcium-dependent chloride conductance mediator pCLCA1. Am J Physiol Cell Physiol 283, C412-C421.

Loewen ME, Smith NK, Hamilton DL, Grahn BH \& Forsyth GW (2003). CLCA protein and chloride transport in canine retinal pigment epithelium. Am J Physiol Cell Physiol 285, C1314-C1321.

Matsuda JJ, Filali MS, Volk KA, Collins MM, Moreland JG \& Lamb FS (2008). Overexpression of CLC-3 in HEK293T cells yields novel currents that are $\mathrm{pH}$ dependent. Am J Physiol Cell Physiol. 294, C251-C262. 
Morris AP (1999). The regulation of epithelial cell cAMP and calcium dependent chloride channels. Adv Pharm 46, 209-251.

Mundhenk L, Alfalah M, Elble RC, Pauli BU, Naim HY \& Gruber AD (2006). Both cleavage products of the mCLCA3 protein are secreted soluble proteins. J Biol Chem 281, 30072-30080.

Nakanishi A, Morita S, Iwashita H, Sagiya Y, Ashida Y, Shirafuji H, Fujisawa Y, Nishimura O \& Fujino M (2001). Role of gob-5 in mucus overproduction and airway hyperresponsiveness in asthma. Proc Natl Acad Sci U S A 98, 5175-5180.

Nilius B, Szucs G, Heinke S, Voets T \& Droogmans G (1997). Multiple types of chloride channels in bovine pulmonary artery endothelial cells. J Vasc Res 34, 220-228.

Patel AC, Brett TJ \& Holtzman MJ(2008). The role of CLCA proteins in inflammatory airway disease. Annu Rev Physiol 71, 425-449.

Piper AS, Greenwood IA \& Large WA (2002). Dual effect of blocking agents on $\mathrm{Ca}^{2+}$-activated $\mathrm{Cl}^{-}$currents in rabbit pulmonary arterial smooth muscle cells. J Physiol 539, 119-131.

Qu Z \& Hartzell HC (2000). Anion permeation in $\mathrm{Ca}^{2+}$-activated $\mathrm{Cl}^{-}$channels. J Gen Physiol 116, 825-844.

Takahashi T, Neher E \& Sakmann B (1987). Rat brain serotonin receptors in Xenopus oocytes are coupled by intracellular calcium to endogenous channels. Proc Natl Acad Sci U S A 84, 5063-5077.
Toda M, Tulic MK, Levitt RC \& Hamid Q (2002). A calcium activated chloride channel $(\mathrm{hClCa} 1)$ is strongly related to IL-9 expression and mucus production in bronchial epithelium of patients with asthma. J Allergy Clin Immunol 109, 246-250.

Wang Q, Wang YX, Yu M \& Kotlikoff MI (1997). $\mathrm{Ca}^{2+}$-activated $\mathrm{Cl}^{-}$currents are activated by metabolic inhibition in rat pulmonary artery smooth muscle cells. Am J Physiol Cell Physiol 273, C520-C530.

Winpenny JP, Lavery WL, Watson N \& Chazot PL (2002). Biochemical and electrophysiological characterization of the GOB5 (mClCa3) chloride channel protein after expression in HEK 293 cells. J Physiol 539, 2P.

\section{Authors Contribution}

All authors participated in the conception and design (M.H.; A.G.; A.J.; J.P.W.; L.P.; K.A.; D.T.; M.M.) or to the analysis and interpretation of data (M.H.; A.G.; N.D.; J.P.W.; L.P.; K.A.; D.T.; M.M.). All authors participated in drafting the manuscript (M.H.; A.G.; N.D.; J.P.W.; L.P.) or revising it critically for important intellectual content (M.H.; N.D.; D.T.; M.M.). All authors have approved the final manuscript. Experiments and data analysis were performed at GlaxoSmithKline Stevenage and data modelling was performed at Leicester University.

\section{Acknowledgments}

This work was supported by GlaxoSmithKline and the Wellcome Trust. 